Pacific Northwest

National Laboratory

Operated by Battelle for the

U.S. Department of Energy

\title{
Chemical Sensing Using Infrared Cavity Enhanced Spectroscopy: Short Wave Infrared Cavity Ring Down Spectroscopy (SWIR CRDS) Sensor
}

\author{
R. M. Williams \\ J.S. Thompson \\ W.W. Harper \\ T.L. Stewart \\ P.M. Aker
}

October 2003

Prepared for the U.S. Department of Energy

under Contract DE-AC06-76RL01830 


\title{
DISCLAIMER
}

This report was prepared as an account of work sponsored by an agency of the United States Government. Neither the United States Government nor any agency thereof, nor Battelle Memorial Institute, nor any of their employees, makes any warranty, express or implied, or assumes any legal liability or responsibility for the accuracy, completeness, or usefulness of any information, apparatus, product, or process disclosed, or represents that its use would not infringe privately owned rights. Reference herein to any specific commercial product, process, or service by trade name, trademark, manufacturer, or otherwise does not necessarily constitute or imply its endorsement, recommendation, or favoring by the United States Government or any agency thereof, or Battelle Memorial Institute. The views and opinions of authors expressed herein do not necessarily state or reflect those of the United States Government or any agency thereof.

\author{
PACIFIC NORTHWEST NATIONAL LABORATORY \\ operated by \\ BATTELLE \\ for the \\ UNITED STATES DEPARTMENT OF ENERGY \\ under Contract DE-AC06-76RL01830
}

Printed in the United States of America

$$
\begin{gathered}
\text { Available to DOE and DOE contractors from the } \\
\text { Office of Scientific and Technical Information, } \\
\text { P.O. Box 62, Oak Ridge, TN 37831-0062; } \\
\text { ph: (865) 576-8401 } \\
\text { fax: }(865) \text { 576-5728 } \\
\text { email: reports@adonis.osti.gov }
\end{gathered}
$$

\author{
Available to the public from the National Technical Information Service, \\ U.S. Department of Commerce, 5285 Port Royal Rd., Springfield, VA 22161 \\ ph: (800) 553-6847 \\ fax: $(703) 605-6900$ \\ email: orders@ntis.fedworld.gov \\ online ordering: http://www.ntis.gov/ordering.htm
}

This document was printed on recycled paper. 


\title{
Chemical Sensing Using Infrared Cavity Enhanced Spectroscopy: Short Wave Infrared Cavity Ring Down Spectroscopy (SWIR CRDS) Sensor
}

\author{
R. M. Williams \\ J. S. Thompson \\ W. W. Harper \\ T. L. Stewart \\ P. M. Aker
}

October 2003

Prepared for

the U.S. Department of Energy

under Contract DE-AC06-76RLO 1830

Pacific Northwest National Laboratory

Richland, Washington 99352 


\section{Summary}

The principal goal of Pacific Northwest National Laboratory's (PNNL's) Remote Spectroscopy Project is to explore and develop the science and technology behind point and stand off infrared (IR) spectroscopic chemical sensors that are needed for detecting weapons proliferation activity and countering terrorism. Missions addressed include detecting chemical, biological, and nuclear weapons and their production; counterterrorism measures that involve screening luggage, personnel, and shipping containers for explosives, firearms, narcotics, chemical weapons and/or their residues; and mapping of contaminated areas. The science and technology developed in this program is dual use in that it additionally supports progress in a diverse set of agendas that include chemical weapons defense programs, air operations activities, emissions monitoring, law enforcement, and medical diagnostics.

Sensors for these missions require extremely low limits of detection because many of the targeted signature species are either present in low concentrations or have extremely low vapor pressures. The sensors also need to be highly selective as the operating environments will contain a variety of interferent species and false positive detection is not an option. PNNL has been working on developing a class of sensors that draw vapor into optical cavities and use laser-based spectroscopy to identify and quantify the vapor chemical content. The cavity enhanced spectroscopies (CES) afford extreme sensitivity, excellent selectivity, noise immunity, and rapid, real-time, in-situ chemical characterization. PNNL's CES program is currently focused on developing two types of sensors. The first one, which is based on cavity ring down spectroscopy (CRDS), uses short wave infrared (SWIR) lasers to interrogate species. The second sensor, which is based on noise immune cavity-enhanced optical heterodyne molecular spectroscopy (NICE OHMS), uses long wave infrared (LWIR) quantum cascade lasers as the light source. This report details the research and discoveries made on the SWIR CRDS project.

While chemical detection limits in the SWIR are not expected to be as low as that in the LWIR, there are a number of reasons for designing sensors that operate in this region. First and foremost is that high quality SWIR lasers, detectors and optics are commercially available. Technological advances made in the telecommunications sector have yielded photonic components that are robust, low power, compact and operate at room temperature. These components can be quickly combined and assembled to produce a sensor prototype. This is exactly what we have done with our cavity ring down sensor. We assembled our first prototype instrument in FY02, tested it in the laboratory, developed the chemometrics, and defined several improvements that needed to be implemented before trialing this sensor in the field. In FY03 we completed the refinements, retested the sensor in the laboratory, and then conducted our first field campaign. Our success was demonstrated by the ability of our SWIR CRDS to run autonomously and continuously for 7 days when located in PNNL's Shipping and Receiving Building. No false positive alarms were detected even though the environment was contaminated with vehicle exhaust fumes, dirt, dust, and volatile organic chemicals associated with packaging materials. The instrument maintained its detection threshold and calibration throughout the test. Small fluctuations that we observed in the background concentration levels have led us to develop a more robust method for calibrating the instrument, and separate tests we conducted in the laboratory have afforded a means to account interference from species that have very broad, but weak absorption in this spectral region. We outline all of these accomplishments in detail in the body of this report. 


\section{Contents}

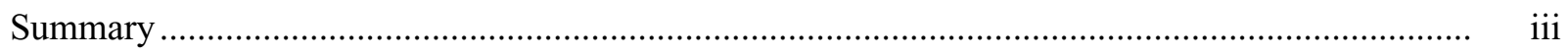

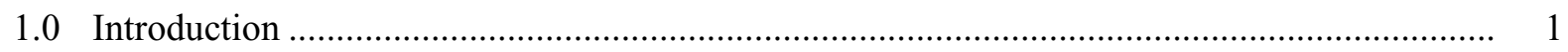

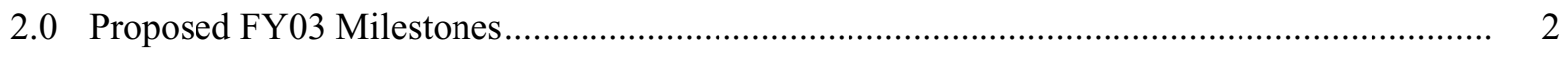

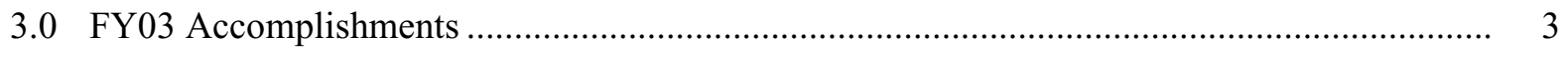

3.1 Sensitivity and Throughput Enhancements ............................................................. 3

3.1.1 CRD Cell Design Considerations ............................................................. 3

3.1.2 Reduced Retention of Active Samples ........................................................... 4

3.1.3 Response Time Improvements ................................................................... 5

3.1.4 Impact of Improved Mirror Reflectivity on CRDS Sensitivity …....................... 7

3.2 Signal Acquisition and Data Processing Improvements ............................................ 8

3.2.1 Implementation of Dual Sampling Schemes .................................................. 8

3.2.2 Reduction in Data Processing Time .............................................................. 9

3.2.3 Autonomous and Continuous Concentration Monitoring ….............................. 10

3.2.4 Improved Concentration Analysis....................................................................... 11

3.3 SWIR CRDS Field Trial .................................................................................... 12

3.3.1 Purpose and Goals of First Field Campaign...................................................... 12

3.3.2 Verification of SWIR Instrument Stability .................................................... 13

3.3.3 Cell Temperature and Pressure.................................................................... 13

3.3.4 Instrument Background Signal and Noise - Cell Optical Integrity ...................... 15

3.3.5 Measurement of Instrument Sensitivity ........................................................ 15

3.3.6 Measurement of Background Variability ....................................................... 17

3.3.7 Conclusion and Future Directions ................................................................... 19

3.4 Feasibility Studies for Sensor Size and Weight Reduction ........................................ 20

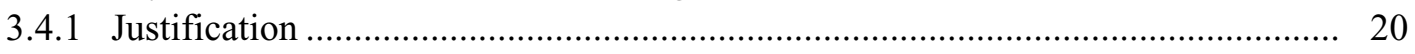

3.4.2 Generation I All Fiber SWIR CRDS …..................................................... 23

3.5 High Resolution SWIR Spectroscopy of WMD-related Molecules............................... 25

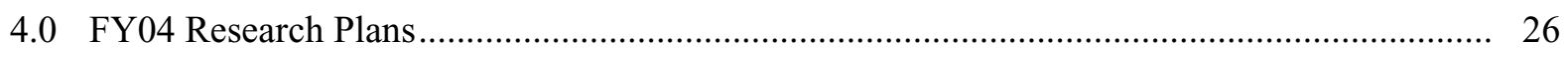

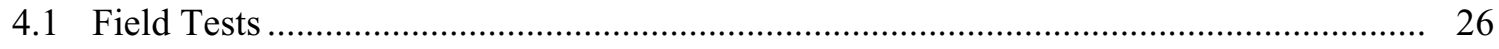

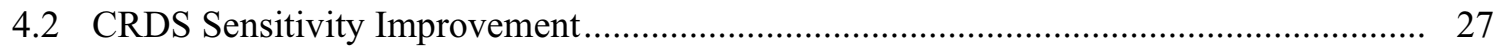

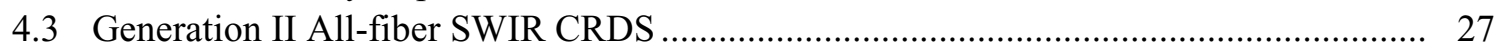

4.4 High Resolution SWIR Spectroscopy of Relevant Molecules …................................. 28

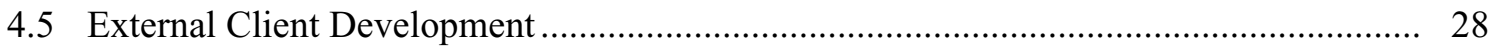




\section{Figures}

1 Schematic of SWIR CRD Optical Sample Cell

2 Results Showing the Observed Ammonia Signals when the Continuous Dilute Ammonia Flow is Quickly Transitioned to Either Dry Air (zero-air) or Ambient Air Using an Unpassivated Optical Cell and a Similar Passivated Optical Cell

3 Time Series Measurements of Ammonia Concentration Obtained with SWIR CRD Instrument. 10

4 Results from a 66-Hour Continuous Operation of PNNL's SWIR CRD Instrument 11

5 Cell Temperature and External Temperature Measured During Field Trial 14

6 Cell Pressure Measured During Field Trial. 14

7 Statistical Analysis of the Ring Down Events During the 7-Day Trial 16

8 Ammonia Mixing Ratio Obtained from Analysis of Recorded Spectra Containing 3 Distinct Absorption Features as Measured Periodically During 7 Day Trial...

9 Series of Chemical Challenges

10 Ammonia Concentration vs. Time for Both the Warehouse and Laboratory Experiments.......

11 Portion of the Ammonia Absorption Spectrum Covered by the Diode Laser

Used in this Work

12 SWIR Fiber-Optic Transmitter Module for Digital Optical Fiber Networking Proposed as Optical Unit For Micro-CRD System.

13 Schematic Representation of SWIR Fiber-Optic Based CRD Instrument

14 Digital Photograph of All-Fiber SWIR CRDS Prototype

15 Early Cavity Ring Down Trace from Fiber CRD Instrument. 25

16 Short-Wave Infrared Absorption Spectrum of Methanol at Low Pressure Using the Modified Bomem FTIR 


\subsection{Introduction}

Since we presented an extensive introduction to cavity ring down spectroscopy (CRDS) in our FY02 final report, we provide here only a brief description of the technique to re-familiarize the reader. CRDS involves measuring optical loss inside a resonator. The simplest optical resonator is made from two highly reflective mirrors that are aligned to support a single optical mode (i.e., a standing wave), which is commonly called a resonance. If the wavelength of a continuous-wave (cw) laser is coincident with a cavity resonance, light intensity will build up because the cavity has a high-Q factor. If the source is suddenly switched off, or tuned off resonance, light will gradually leak out of the cavity due to the slight transmission of each mirror $(<0.1 \%)$ and other diffractive and/or absorptive losses. The decay phenomenon is termed "ring down" as it mimics how the sound from a finely-tuned, or resonant, bell slowly decays after it is struck. Mathematically, this ring down can be expressed as an exponential decay curve with an associated ring down time constant.

When the cavity is empty the terms governing the temporal evolution of the decay transient can be defined as,

$$
i(t)=i_{o} \exp \left[-(1-R) \frac{t c}{L}\right]
$$

where $\mathrm{c}$ is the speed of light, $\mathrm{R}$ is the mirror reflectivity, $\mathrm{L}$ is the cavity length, and $\mathrm{t}$ is time. The decay time constant (i.e., the time when $i$ equals $1 /$ e of $\mathrm{i}_{0}$ ) is given by,

$$
\tau_{\text {empty }}=\frac{L}{c(1-R)} .
$$

If an absorbing species is added to the empty cavity, it reduces the ring down time, i.e.

$$
\tau_{\text {total }}=\frac{L}{c[(1-R)+\alpha L]},
$$

where $\alpha$ is the absorption coefficient in units of $\mathrm{cm}^{-1}$. The absorption coefficient is calculated by comparing the ring down times associated with an empty and a filled cavity, i.e.

$$
\alpha=\frac{1}{c}\left[\frac{1}{\tau_{\text {total }}}-\frac{1}{\tau_{\text {empty }}}\right] .
$$

The absorption coefficient is related to the species concentration via $\alpha=\sigma \mathrm{N}$, where $\sigma$ is the molecular absorption cross section in $\mathrm{cm}^{2} /$ molecule and $\mathrm{N}$ is the number density, or concentration, in molecule $/ \mathrm{cm}^{3}$. 


\subsection{Proposed FY03 Milestones}

The SWIR CRDS sensor, initially assembled in FY02, is an automated field experiment consisting of the optical sensor plus subsystems for air sampling and handling, sample purging, calibration, data acquisition, and data processing. It is installed in a trunk-sized shipping container for transportation to field sites and protection during experiments.

Our first generation SWIR CRDS is designed to detect ammonia. This species was chosen because it is "sticky" and can be highly reactive under certain conditions. Many explosives, chemical warfare agents (CWAs) and related compounds have these same properties, and so any sensor designed to detect and quantify them must have inlet and sampling system walls that reduce, or at least mitigate, the effect that sticking and reaction have on quantitative analysis (chemometrics).

Our proposed FY03 research plan for the SWIR CRD project at PNNL addressed several issues associated with improving the sensor performance and moving it into the field. Some of the specific topics included:

- Sensor Sensitivity and Throughput Enhancement

- Signal Acquisition and Data Processing Improvements

- SWIR CRDS Lab and Field Tests to Assess the Impact of Interferents

- Feasibility Studies for Sensor Size and Weight Reduction

- High Resolution SWIR Spectroscopy of WMD-related Molecules.

Significant progress was made in all five of these research areas and in most cases all of the proposed goals were met. In brief:

- Ammonia retention (a chemically active gas) in the sample cell was reduced by passivating the optical cell with a glass-like coating.

- Instrument response time was reduced from $\sim 85$ seconds to as little as 3 seconds for ammonia.

- Software improvements resulted in a 5-fold improvement in ring down signal processing time and provided higher flexibility in detection approaches.

- New software features were implemented so that continuous monitoring could be done.

- An electrical and gas-handling umbilical interconnect (which interfaces the instrument support rack and the portable optical bench) was constructed. This allowed the sensor to be moved out of the laboratory and into the field.

- The first field trials were completed. 
- The performance of a smaller, all fiber SWIR CRD prototype is under investigation.

- The SWIR spectroscopy of chemical compounds related to WMD proliferation is being explored, both experimentally and through literature investigation.

The following sections more thoroughly describe the specific improvements made to the system.

\subsection{FY03 Accomplishments}

\subsection{Sensitivity and Throughput Enhancements}

\subsubsection{CRD Cell Design Considerations}

Many technical challenges need to be addressed in the design of a functional CRD sample cell. This includes cell volume, cell length, vacuum integrity, gas flow conductance, chemical compatibility, operating temperature and stability, and cavity mirror scanning mechanism. As is the case with other analytical instrumentation, a single design is not optimal for all sampling scenarios. In our design considerations we focused on a deployment scenario which involves drawing large volumes of ambient air through the instrument to provide real-time analyte concentration measurements. A schematic of our current CRD optical cell is shown in Figure 1.

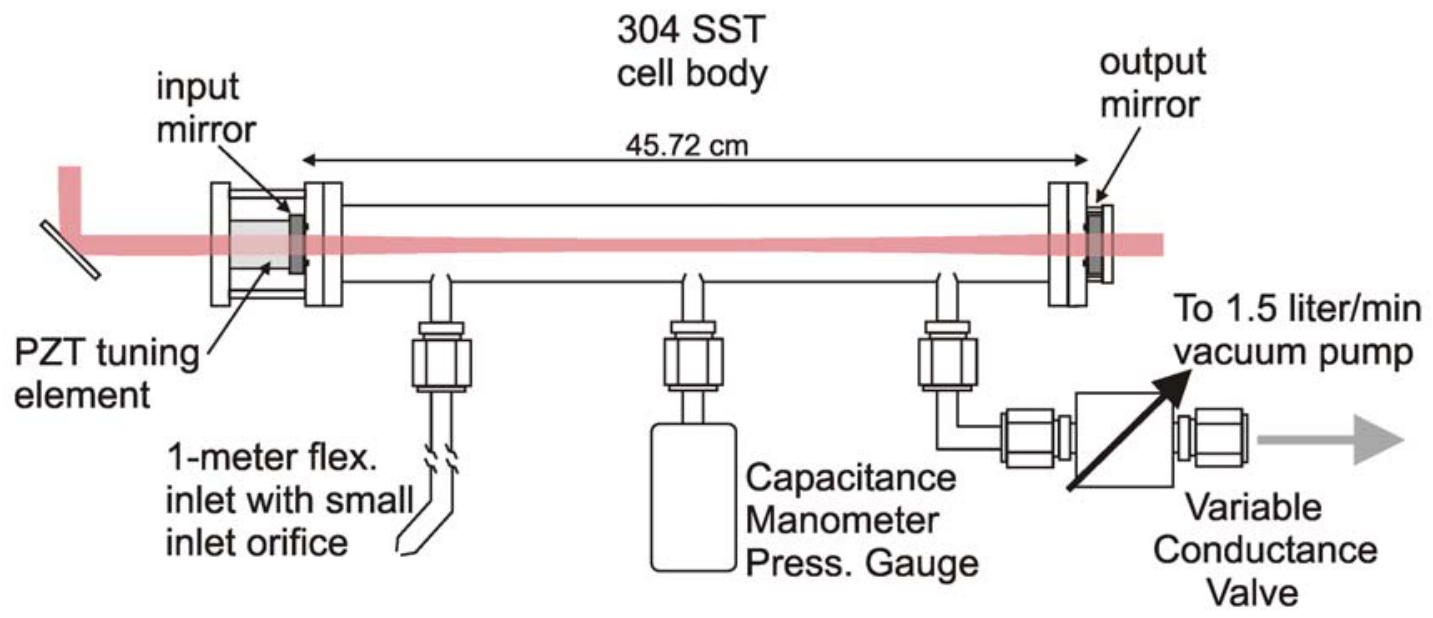

Figure 1. Schematic of SWIR CRD Optical Sample Cell

The two major improvements in cell design over the previous unit are: (1) the addition of a passivation coating for decreased retention of reactive compounds and (2) larger inlet and outlet ports resulting in greater throughput and faster response times. The following section is a brief description of the current sample cell. 
The cell consists of a stainless steel (304 SST) tube with a length of $45.72 \mathrm{~cm}$ fitted with standard ultra-high vacuum flanges. The inner diameter is $3.8 \mathrm{~cm}$ yielding a cell volume of 0.52 liters. The high reflecting mirrors are pressed against Viton o-rings forming a vacuum seal while allowing for fine adjustment of cavity alignment. A displacement force is applied to the air-side of the input mirror using a piezoelectric tube transducer (PZT) capable of linear extension of $\sim 10$ microns. As the PZT's length is changed (with the application of high voltage) the input mirror is pressed against the flexible o-ring allowing the separation between the two mirrors to be continuously varied. Three vacuum fittings provide access for a gas inlet, a vacuum pump-out port, and a pressure monitor. Cell pressure is measured with a temperature stabilized capacitance manometer, which provides an accuracy of $0.1 \%$ (independent of gas composition).

Consumable gases (i.e., calibrant gas and zero-air gas) are introduced through a pair of dedicated mass-flow controllers (offering flows up to $100 \mathrm{std}$. cc/min) while ambient samples are drawn through (and controlled by) a $10000 \mathrm{std}$. cc/min mass flow controller. Gas pumping is achieved by the use of a dry-diaphragm vacuum pump by itself (for high throughput), or in combination with a small turbo molecular pump (lower throughput). To better control the operating pressure of the CRD instrument an inline variable conductance valve (VCV) is placed near the cell. The VCV is an important piece of the system because it allows the instrument to be operated under a variety of pumping modes. This is especially useful during system calibration when a small amount of a dilute gas is continuously flowed through the system while a spectrum is recorded. Without the VCV a very large volume of calibration gas would be needed - meaning that a large cylinder would have to accompany the instrument in the field. With the VCV the same calibration routine can be accomplished with a small portable cylinder mounted inside the instrument support rack.

The exterior of the cell is wrapped with foil heaters which are capable of heating the sample body to $\sim 100^{\circ} \mathrm{C}$ with an accuracy of $\pm 0.2^{\circ} \mathrm{C}$ (further stabilization is not necessary for CRD detection). Currently, the cell body is held at approximately $35^{\circ} \mathrm{C}$ in order to lessen the likelihood of water adsorption on the inner surface of the chamber. The CRD cell body was coated by a 3rd party vendor (Restek Corp) which specializes in chemically resistant coatings for analytical instrumentation. The proprietary coating is 120 -nm thick and is based on fused silica (i.e., ordinary glass).

\subsubsection{Reduced Retention of Active Samples}

As mentioned in the FY02 final report, the SWIR CRD instrument is being designed to detect a variety of chemicals associated with WMD. Many of these chemicals are either sticky (i.e., have low volatility) or undergo surface catalyzed breakdown. Ammonia is another molecule that exhibits these properties. Based on this consideration, and given its well known spectroscopy, this species was selected as our target molecule. Detection of ammonia has proven to be challenging. In FY02 we discovered that it would "stick" to the inner surfaces of the CRD cell, thus producing errors in the measured concentration levels. Of additional interest was the sudden increase (followed by a steady decrease) in detected ammonia concentrations when ambient air was introduced into the cell after an ammonia dilution had been present. This ammonia spike was attributed to the presence of water in the ambient samples which facilitates ammonia desorption from the interior metal surfaces. This was confirmed by controlled tests 
with and without dry air. The dry air produced no ammonia spike. Figure 2 presents the results from this test.

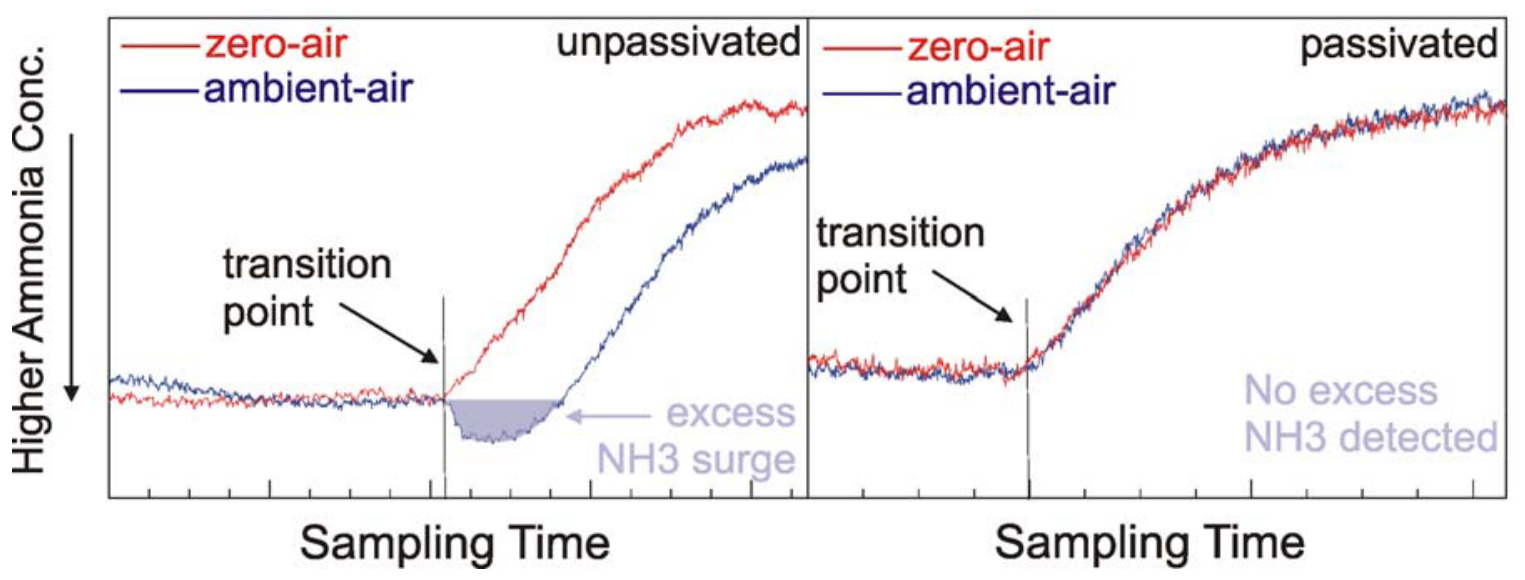

Figure 2. Results Showing the Observed Ammonia Signals when the Continuous Dilute Ammonia Flow is Quickly Transitioned to Either Dry Air (zero-air) or Ambient Air Using an Unpassivated Optical Cell (left panel) and a Similar Passivated Optical Cell (right panel). The entire time duration for either test is 350 seconds. Excess ammonia is observed when transitioning to ambient air using the untreated cell, while no excess ammonia is observed with the treated cell.

The left panel in Figure 2 compares how the original optical cell responds to a change in ammonia concentration. As the inlet is transitioned from the dilute ammonia sample (50 ppmv) to room air there is a notable increase in the observed ammonia signal which persists for approximately 30 seconds. Ammonia concentration then decreases at the same rate observed for dry air. The panel on the right in Figure 2 shows the results from the same test except with the use of the chemically passivated cell (silica coating). No noticeable increase in ammonia signal is observed. The conclusion from the test is that ammonia is no longer being retained on the interior surface of the optical cell. This result suggests that the silica coating may be a critical performance enhancing factor in the design of optical sampling sensors, providing higher accuracy in the observed analyte concentration.

\subsubsection{Response Time Improvements}

Optimizing the response time of an optical sampling sensor is extremely important and depends critically on the deployment strategy of the sensor. The PNNL SWIR CRDS instrument is being optimized for rapid sampling consistent with either baggage or facilities inspection. A discussion of deployment strategies is given in more detail below. In order to optimize the response time of the instrument several key factors need to be understood and optimized. Those which have been addressed in FY03 include higher gas flow capacity through the optical cell, and optimization of the data acquisition and data processing routines. 
Gas throughput in an optical sampling sensor is related to the net pumping speed of the vacuum system on the outlet side of the sensor and the throughput of the sampling head on the inlet side of the sensor. Components for the gas handling system are selected based on the desired operating pressure of the sampling unit; in this case 50-100 Torr was chosen. This pressure allows maximum analyte concentration to be present in the cell while still reducing the pressure broadening effect allowing for maximum chemical selectivity. The previous optical cell incorporated a smaller variable conductance valve on the outlet side which limited the flow rate through the sample cell to $100 \mathrm{std}$. cc/min (SCCM). This smaller VCV was chosen to match the flow rates of the inlet controllers to produce controlled pressures in the 10-100 Torr range. Based on basic vacuum calculations, the net pumping speed, $\mathrm{S}_{\text {net }}$ (liter $\mathrm{sec}^{-1}$ ) can be obtained using,

$$
S_{\text {net }}\left(\text { liter } \mathrm{sec}^{-1}\right)=Q\left(\text { torr liter } \mathrm{sec}^{-1}\right) / P(\text { torr })
$$

where $\mathrm{Q}$ is the gas throughput of the system and $\mathrm{P}$ is the measured pressure of the system. When flowing at $18 \mathrm{SCCM}\left(0.23\right.$ Torr liter $\left.\mathrm{sec}^{-1}\right)$ the pressure in the original optical cell was 60 Torr, resulting from the net pumping speed of 0.004 liter $\mathrm{sec}^{-1}$. This was well below the net pumping speed of the vacuum pump and connecting lines calculated to be 1.4 liter $\mathrm{sec}^{-1}$. A higher throughput (20000 SCCM) VCV replaced the smaller unit. This combination resulted in a net pumping speed of $\sim 1$ liter $\sec ^{-1}$ allowing inlet flow rates of up to $10000 \mathrm{SCCM}$ while maintaining a continuous operating pressure of 60 Torr. The increased pumping speed dramatically improves the sensor response time. The time required to exchange the gas within the cell is given by $\mathrm{V}_{\text {cell }} / \mathrm{S}_{\text {net. }}$. Since $\mathrm{V}_{\text {cell }}=0.52$ liters and $\mathrm{S}_{\text {net }}=1$ liter $\mathrm{sec}^{-1}$, the exchange time is $0.52 \mathrm{sec}$, a significant improvement over our previous design which had an exchange time of $130 \mathrm{sec}$.

Despite the dramatic increase in pumping speed there is one significant drawback to such a high conductance, the rate of consumption of calibration and zero-air gases will scale with the pumping speed. With our previous arrangement, a small portable cylinder of calibration gas (105 liters) would last an estimated 100 hours of continuous operation at a flow rate of 18 SCCM (although the response time would be limited to 130 seconds). The present high-flow configuration results in very high throughput; however, the contents of an entire cylinder would be used in 21 minutes at a flow rate of 5000 SCCM. To allow for the use of small portable cylinders of calibration gas and zero-air a dual outlet system was integrated into the system. When sampling ambient air, the high conductance outlet valve is opened to allow rapid response to changes in analyte concentration. During a calibration or background cycle the high conductance valve is closed and the smaller conductance valve "throttles" the throughput of the system, thus minimizing usage of consumable gases. While the larger valve does have a variable flow rate, its minimum controllable range is still far beyond what is needed for reduced flow.

Equally important to the response time of the cell is the design of the inlet systems. Our original inlet design used three mass flow controllers (MFC): ambient, calibration gas and zero-air (each with a maximum flow rate of $100 \mathrm{SCCM}$ ). It was envisioned that ambient samples would be introduced into the cell and analyzed for a fixed period of time then the MFCs would cycle to the calibration gas to ensure system operation and finally cycled to zero-air (i.e., ultra-pure dry air) for a baseline measurement. Time was spent ensuring that these transitions could be made without changes to the operating pressure of the optical cavity. It was thought that a sudden pressure change could misalign the optical cavity and cause 
instabilities within the system. Testing however showed that the cavity alignment is insensitive to large pressure changes in the range of 0.1 to 200 Torr occurring in short bursts $(<5 \mathrm{sec})$. Pressure changes do cause the cavity length to vary, however this effect positively augments the deliberate cavity length modulation accomplished with the use of the PZT scanner.

In order to take advantage of the increased pumping speed at the cell outlet, an inlet with a comparable flow rate is necessary. The original ambient sampling MFC had a maximum flow rate of 100 SCCM producing a steady-state pressure in the optical cell of 1 Torr (too low for optimal sensing) with the larger outlet VCV in place. Two high-conductance inputs are now available for use with the system; the first being a higher (10000 std. cc/min) MFC and the second being a simple "sampling wand". Using the sampling wand the ambient sampling MFC is by-passed using a simple manual on/off vacuum valve with $\sim 1$ meter of flexible $1 / 4$ " OD polyethylene tubing. At the end of the tubing we attached a short section of copper tube pressed in a vise to produce a thin slit which acts as the system inlet. This approach produced a steady-state cell pressure of 57 Torr, corresponding to a flow rate through the slit of 4500 SCCM (or 4.5 standard liters per minute). The sampling wand is flexible and easy to manipulate. Once the vapor sample is drawn through the small inlet orifice, it is rapidly transported to the optical cell; as opposed to a design where the flow rate is controlled in mid-stream, in which case, the inlet air sample would move slowly down the lead portion of the tube then finally into the cell, resulting in a degradation of the response time. The remaining two MFCs for the calibration gas and the zero-air gas were reintegrated into the system using a dual inlet design that allows for high flow rates of ambient samples and reduced flow rates for consumables. With these optimized flow rates, the response time of the system was no longer limited by gas transport but by the time needed for processing the data.

\subsubsection{Impact of Improved Mirror Reflectivity on CRDS Sensitivity}

One modification which can be made to improve the detection limit of any cavity ringdown instrument is to use cavity mirrors with higher reflectivity. We estimate the reflectivity of our current mirrors is approximately $99.93 \%$. This is an approximate value established by measuring the actual cavity ringdown time constant with an empty cavity and ignoring any secondary loss effects such as diffraction loss of the TEM00 mode in the resonator. Because of the high reflectivity, the effective pathlength probed by the laser is $>1.3 \mathrm{~km}$ in a physical cell of length 0.47 meters. By increasing the mirror reflectivity to $99.99 \%$ the effective pathlength grows to approximately $10 \mathrm{~km}$, theoretically yielding an improvement in detection limit of nearly a factor of ten. While such mirrors are commercially available (and we have tried them in our system) several key factors preclude the easy transition to higher reflectivity mirrors.

To first order, if the reflectivity of the mirrors is increased the observed transmission intensity through the cavity decreases resulting in a lower signal to noise ringdown transient. Even though the signal-contrast will increase with mirror reflectivity, the overall signal level itself decreases. The second factor which is particularly important to $\mathrm{cw}$-cavity ringdown spectroscopy is the ratio between the laser linewidth and the cavity linewidth. With higher reflectivity mirrors the cavity finesse increases, hence the spectral width of each individual resonance feature decreases. Presently the cavity resonance has an estimated width of $75 \mathrm{kHz}$, while the laser linewidth (measured at short times) is roughly the same order of magnitude as the cavity linewidth. When going to the higher reflectivity mirrors the cavity linewidth is 
reduced to $10 \mathrm{kHz}$ (five to ten times smaller than the laser linewidth). The consequence of this mis-match is a reduction in the injection efficiency of the laser into the cavity by virtue of the fact that the laser experiences larger frequency deviations than the actual width of the cavity resonance during the short amount of time the two are spectrally coincident. These two factors (reduced transmission and laser phase noise effects) limit the benefits of higher finesse cavities in CRDS. Despite the clear technical challenges of going to a CRDS system with higher reflectivity mirrors we believe that a real improvement in detection limit can be realized with some modification to the existing system. These improvements consist of actively controlling the laser's frequency to maintain the required resonance condition long enough to achieve good injection efficiency.

\subsection{Signal Acquisition and Data Processing Improvements}

Using the previous optical cell design the rate-limiting factor in signal response time was a combination of gas retention and gas throughput. Therefore, only a basic signal acquisition and processing software module was needed. Once improvements in gas handling were made a second more advanced signal processing system was implemented. In order to explain these improvements to the overall CRD software module, the basic data acquisition sequence needs to be outlined.

\subsubsection{Implementation of Dual Sampling Schemes}

Presently, the instrument can operate in one of two modes from which analyte concentration can be obtained. In the first mode, an absorption spectrum is recorded over a finite wavelength interval (typically $0.3 \mathrm{~nm}$ wide). The analyte absorption features within the spectrum are fit using a non-linear fitting routine to obtain the integrated absorbance under each feature which is then used to calculate the analyte number density within the cell using the temperature corrected line strength for each transition. The analyte mixing ratio (e.g., ppmv) is then obtained using the known cell pressure (i.e., the total number in the sample cell). To get an absorption spectrum, the laser is moved to a specific wavelength and a fixed number of exponential ring down events are recorded, averaged and fit to obtain the ring down time-constant. This time-constant is stored along with the laser wavelength before the laser is moved to the next specified wavelength where the process is repeated. The final result is a "timeconstant" spectrum which is easily converted to an absorption spectrum (see FY02 final report for specifics). A typical spectrum may consist of 200 wavelength steps (over the $0.3 \mathrm{~nm}$ span) with each data point resulting in 20-50 averaged ring down events. Presently, individual ring down events occur at a rate of $180 \mathrm{~Hz}$.

The second method for data acquisition involves scanning the laser to the peak of an analyte absorption feature (usually done with the use of a reference gas cell containing the analyte) and recording a series of ring down time-constants. Periodically, the wavelength is tuned to a region with no analyte absorption to obtain a baseline measurement that is used to determine absolute concentration. This technique requires the laser's wavelength to be stable over the measurement cycle, which has not been an issue at this time.

The two different techniques have their strengths and weaknesses. Recording an entire absorption spectrum takes longer (15-20 seconds); however since more spectral information is available higher 
sensitivity and higher confidence in the final analyte concentration results. Rapid sampling on the analyte peak provides for a dramatic improvement in response time (e.g., better than 1 concentration

measurement per second); however, less information is available. We have discovered that a hybrid is the best solution. Here the user uses the wand to sense the air around various objects using the rapid sampling mode. When signal above some preset level is detected, the instrument switches modes and records an entire absorption spectrum to confirm or deny the analyte presence. In either mode of operation, optimal signal processing is key to efficient use of the sensor.

\subsubsection{Reduction in Data Processing Time}

Originally, the entire instrument interface was written in a programming environment known as LabView (National Instruments Corp.). One very nice feature of this software is that it allows the developer to run a benchmark routine which analyzes the software and reports exactly how much time was spent within each subroutine. This analysis was first performed within the acquisition mode where the entire spectrum is recorded. We discovered that an enormous amount of time was being spent within the non-linear fitting routine used to fit the absorption profiles. The computationally intensive non-linear method used for this is the well known Levenberg-Marquardt fitting routine. The method is so popular that National Instruments supplies a Levenberg-Marquardt (LM) subroutine for use within their software. It was concluded that while the LabView LM routine was simple to use it was very slow. It was therefore replaced with a custom LM fitting routine written in C. The substitution dramatically increased the speed of fitting the line profiles of the absorption features, even when a more complex Voigt line shape is used.

The next improvement that was made involved more efficient use of data acquisition hardware. The first generation data acquisition system transferred each individual ring down transient curve from the analog-to-digital conversion (ADC) board in the personal computer to the computer's system memory. This transfer process resulted in dead-time while data transfer was occurring. Our current version of the data acquisition system acquires and stores all ring down transients for each wavelength step directly on the ADC board (which has 1 Mbyte of onboard memory). After a specified number of events have been stored, the data block is transferred only once. This multiple-record process results in no lost ring down events during a specific acquisition cycle.

The final improvement made during FY03 was the integration of the custom C Levenberg-Marquardt routine into the fitting procedure used to extract the exponential time-constant of the averaged ring down trace. Once integrated, these improvements lead to a factor of 5 reduction in the data acquisition and signal processing time needed to record a spectrum and compute an analyte concentration. The new system was tested by recording a $0.2 \mathrm{~nm}$ wide spectrum of ammonia near $1531.7 \mathrm{~nm}$ which consists of two well defined features. Spectra consisting of 100 wavelength steps and 10 ring down events averaged per wavelength step were recorded at a rate of 3.1 spectra per minute ( $\sim 19$ seconds/spectrum). Even more impressive is the rate of data collection in the "rapid sampling" mode when the laser is fixed on the absorption peak and individual time-constants are plotted in rapid succession. In this mode, 10 transients are acquired, averaged and fit every $0.2 \mathrm{sec}$. ( 5 data points/sec). The results of a recent 5 minute test are shown in Figure 3 in which the rapid sampling technique was used. 


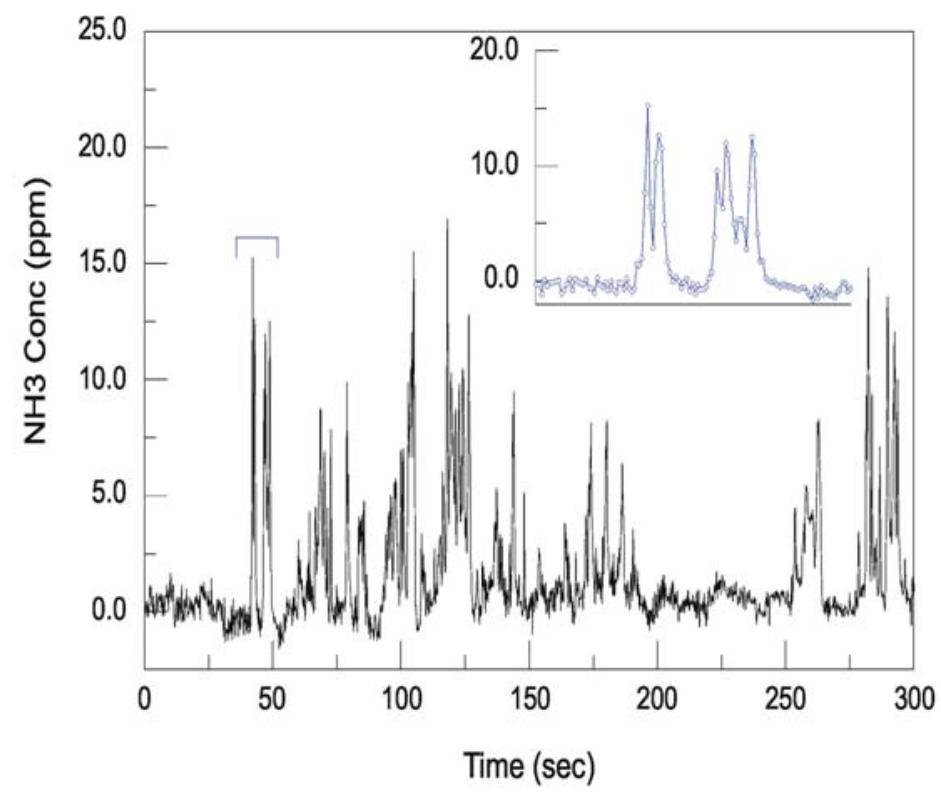

Figure 3. Time Series Measurements of Ammonia Concentration Obtained with SWIR CRD Instrument. A 1-meter flexible sampling wand was used to draw room-air (containing ammonia) into the optical cell at a flow rate of 4500 SCCM. Data points are collected at a rate of $5 \mathrm{~Hz}$. The observed gas exchange rate is 1-2 sec (i.e., the entire gas volume of sensor is exchange in that time period). The inset shows an expanded region between 35 and 55 seconds.

In the test shown in Figure 3 the sampling wand was moved over an open beaker that contained a few drops of Windex. It was noted in the FY02 final report that the vapor pressure of ammonia over window cleaner is $\sim 18$ Torr, which rapidly dilutes under ambient conditions. From the data in Figure 3 a gas exchange rate of 1-2 sec is deduced from the rapid change in signal magnitude. Operating in this mode the signal processing software operates at a rate faster than the gas exchange rate. Higher signal-to-noise data could be obtained at a slower data rate (e.g., $2 \mathrm{~Hz}$ versus the current $5 \mathrm{~Hz}$ ) while still being essentially limited by the gas handling system. Just prior to, and immediately after, the data was taken for Figure 3 the laser was manually tuned off resonance to record a value for the baseline which remained constant (another indication that the passivation coating reduces ammonia retention). This baseline checking can be added as an automated feature in the software interface (see section on interferent suppression).

\subsubsection{Autonomous and Continuous Concentration Monitoring}

In addition to improvements made to speed up data acquisition, enhancements were incorporated into the fitting routines that have allowed the SWIR CRD instrument to log analyte concentrations autonomously and continuously. Figure 4 shows the results from one extended sampling test in which water vapor was monitored. Additional improvements to the software allow continuous monitoring for compounds that may suddenly appear (a more likely deployment scenario). For the data shown in 
Figure 4, water concentration measurements were logged every 40 seconds. In the narrow wavelength range scanned by the instrument $(0.3 \mathrm{~nm})$ two water absorption features are distinct and easily observable (lines a and b shown in Figure 4). While the 66-hour concentration trends for the two water lines track very well, there is a slight discrepancy between the two observed mixing ratios. Visual inspection of the actual absorption spectra did not reveal any problems with the fitting routine which fit both the line shapes and baseline well. We suspect that the $10-15 \%$ offset in the concentrations (and mixing ratios) is possibly due to an error in the published line strengths for the two transitions. Line strengths used to compute absolute concentration were taken from the HITRAN 2002 database. Obtaining a precision and accuracy of individual line strengths to the $10 \%$ level is considered good by most standards, which is why the HITRAN database is so valuable to spectroscopists. However, the molecules most relevant to nonproliferation detection are not contained in the database.

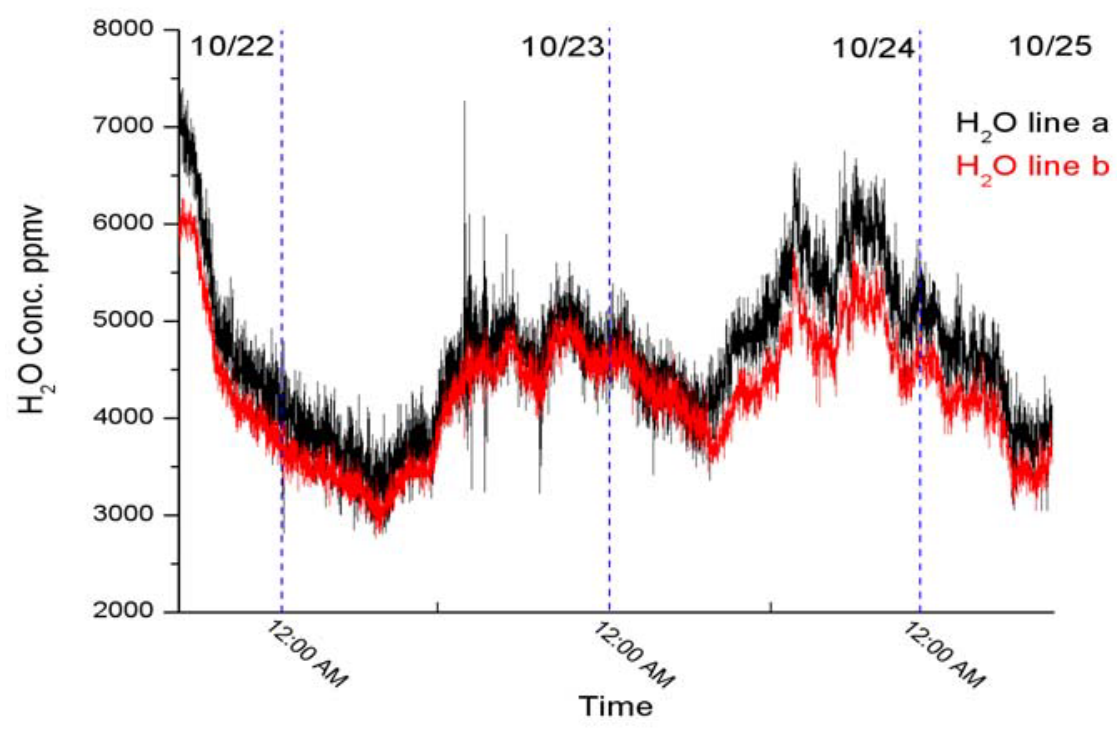

Figure 4. Results from a 66-Hour Continuous Operation of PNNL's SWIR CRD Instrument. The instrument was configured to monitor water vapor concentration during the test by measuring and fitting two distinct water absorption features near $1531 \mathrm{~nm}$.

\subsubsection{Improved Concentration Analysis}

Analyte concentration is established via chemometric analysis of the observed absorption spectrum. This involves fitting the absorption features to an appropriate line shape, followed by integration of each line profile and finally calculating the concentration using the integrated absorbance and the known line strength for the observed transition. The analysis has been further refined and improved by using the simultaneously recorded analyte reference spectrum as a fitting guide. Normally for each observed peak in the CRD spectrum there would be a total of four parameters used in the fitting routine: peak height, peak center location, Gaussian width and Lorentzian width. Both Gaussian and Lorentzian widths are required because the apparatus is operated at a pressure where the Lorentzian pressure broadened component is roughly the same size as the natural Doppler component (i.e., the Gaussian width). By recording an analyte reference spectrum, the peak center positions can be directly read and sent to the 
fitting algorithm thereby reducing the number of parameters needed for the fit and subsequently improving the overall performance of the algorithm. Additionally, the recorded analyte reference spectrum can be used to isolate and quantify only analyte peaks in an observed spectrum when multiple peaks from different absorbers are present.

\subsection{SWIR CRDS Field Trial}

\subsubsection{Purpose and Goals of First Field Campaign}

There were two main objectives for our first short wave infrared (SWIR) cavity ring down spectroscopy (CRDS) sensor field experiment. The first was to verify that the instrument, when operated in a non-laboratory environment, could operate stably and reproducibly over a long period of time. The second was to quantify the background variability (i.e., determine the effect that species other than ammonia have on the signal at the wavelengths for ammonia detection) that results when sensing experiments are conducted in a relatively dirty environment. We conducted our first field experiment at the PNNL shipping and receiving building (790 6th Street) over a period of 9 days. This particular location was chosen because the residual background $\mathrm{NH}_{3}$ level $(<10 \mathrm{ppbv})$ is not detectable by our instrument (minimum detectivity of $200 \mathrm{ppbv}$ ), but there are a sufficient number of different pollutants, such as NOx and VOC species and airborne particulates, to enable us to determine the influence that these contaminants have on background variability. It is important that we be able to quantify background variability in a number of different settings as signals that result from interfering species may give a false positive alarm.

Prior to conducting experiments in the field, we performed identical experiments in the laboratory. Our reasons for doing so were two-fold. First, a practice run through the experimental measurement protocol alerted us to unanticipated problems, and provided us with a precise measure of the experimental timeline. Second, information we collected on background variability and instrument drift was used to characterize the instrument performance (which includes things such as low variance in measured concentration values, limit of detection, rapid and consistent time response, and invariable background noise) in a relatively clean environment.

For most of the experimental time the sensor was operated in the fast data acquisition mode, which we call real-time, or line, monitoring. In this arrangement, the laser wavelength is set to coincide with a peak absorption feature of the gas that is present in the reference cell, which for this field trial was ammonia. The laser wavelength was kept fixed as successive ring down events were captured, averaged, and fit to yield a time constant. The time constant was plotted and logged at a fairly high rate of 5 points per second. By itself, the time constant cannot be used to compute the absorbance. Just as with normal absorption spectroscopy there must be a comparison with a baseline.

The baseline ring down value is acquired by recording the ring down constant when the laser's wavelength is tuned off the resonant ammonia peak to a pre-specified position in the baseline where no ammonia absorption occurs (determined in a prior experiment). The sample absorbance, concentration, and mixing ratio is then determined by comparing the sample ring down time with that of the baseline. 
While real-time monitoring affords a rapid assessment of the local environment, it cannot distinguish between absorption by the analyte or by another interferent species. Since part of our mission was to identify species that could give rise to a false positive signal, we preprogrammed the sensor to switch from line monitoring mode to spectrum monitoring mode when the signal reached a critical absorbance value. If ammonia was the source of the signal, this procedure would provide positive confirmation. If not the spectrum could be used to identify, or at least provide some clues, as to the interferents identity.

The SWIR CRDS was programmed to run through a calibration and performance self-check routine every 1.5 hours so that data could be collected on sensitivity and stability drift. The procedure first involved passing a zero-air gas sample through the cavity and measuring the ring down time associated with the blank sample. (Drifts in the zero-air ring down times can indicate that the cavity optics are being contaminated by dirt or reactive species.) After this a calibrant ammonia mixture (50 ppmv $\mathrm{NH}_{3}$ in nitrogen) was passed through the cell and the concentration determined and logged. Long-term degradation of this value would be an indication of sensitivity drift. The system was then flushed with zero-air to remove any residual ammonia from the optical cavity and the sensor was then restarted in line monitoring mode with ambient air flowing through the instrument.

\subsubsection{Verification of SWIR Instrument Stability}

Instrument instability may give a false background signal, and so it is important that we monitor this concomitant with measuring background variability. A number of parameters may contribute to instrument instability including drifts in the instrument's baseline ring down time and signal to noise ratio (SNR), and drifts in the instrument's sensitivity. Drift in these parameters may be caused, in part, by optical misalignment due to temperature and pressure fluctuations, and contaminant and dirt collection in the ring down cavity.

\subsubsection{Cell Temperature and Pressure}

Results from our field experiment show that external temperature fluctuations do not affect our instrument response, primarily because we heat the cavity ring down cell to a constant value of $\sim 317 \pm$ $0.5 \mathrm{~K}$. Figure 5 shows a plot of the external temperature and internal cell temperature measured over a 7 day period. While the external temperature fluctuates by as much as $6{ }^{\circ} \mathrm{C}$, the internal temperature remains constant, within $\pm 0.5^{\circ} \mathrm{C}$. Our results also show that the cell pressure remains constant over an extended period. As Figure 6 shows, pressure variations of only \pm 0.25 Torr are observed when the system is configured to maintain the cell pressure at 50.2 Torr. 


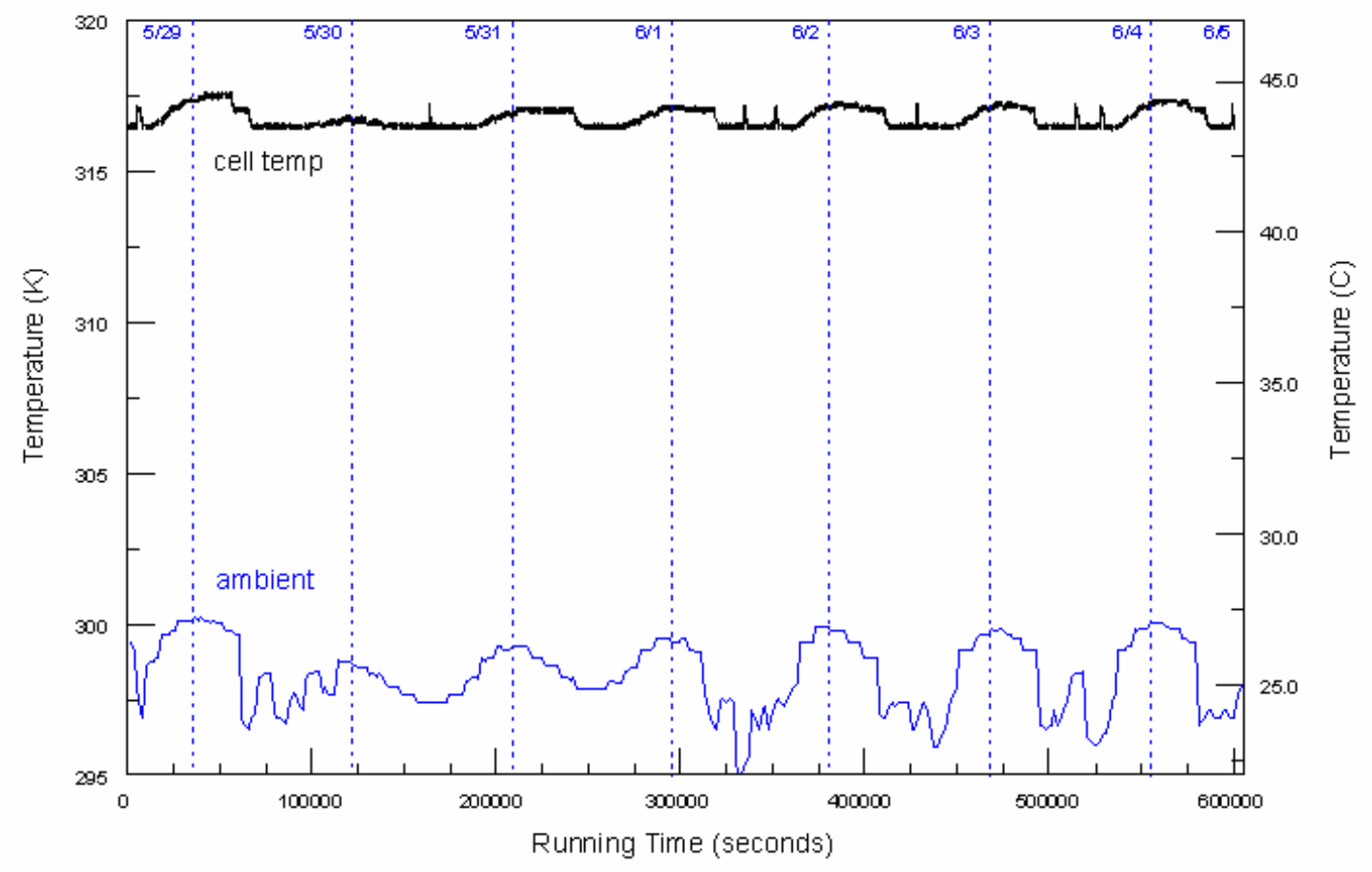

Figure 5. Cell Temperature and External Temperature Measured During Field Trial

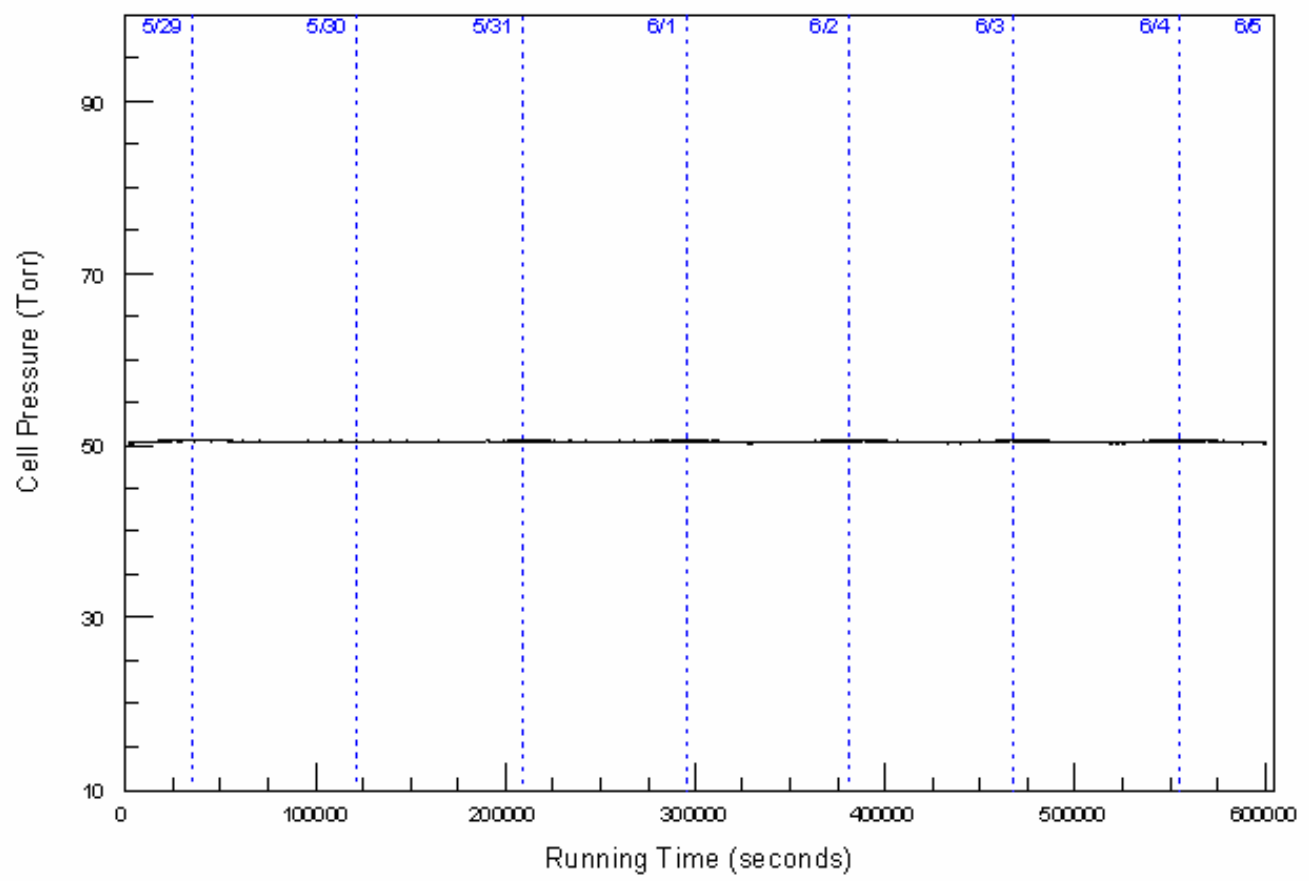

Figure 6. Cell Pressure Measured During Field Trial 


\subsubsection{Instrument Background Signal and Noise - Cell Optical Integrity}

In a clean laboratory environment our instrument noise hovers around $0.5 \%$ of the baseline. This is a result of the sampling jitter on the cavity ring down curves. To confirm that this experimental parameter is independent of operating environment, every 1.5 hours we measured a zero air sample spectrum and determined the background ring down time and jitter in several spectral windows.

While we did not, a priori, expect particulate accumulation on the cavity mirrors to be a significant source of instrument instability because we installed a filter on the inlet system, we did need to prove that the filter is adequate enough for this particular dirty environment. Any condensation or chemical reaction of gas phase species on the cavity mirrors will be reflected by a change in the cavity ring down time when a zero air sample is present in the chamber. Figures $7 \mathrm{a}, 7 \mathrm{~b}$, and $7 \mathrm{c}$ plot a series of cavity ring down times, their standard deviations and the relative error of each measurement, respectively. Each measurement was performed every 120 minutes, using a zero-air sample during the 7-day test. As can be seen from the Figure $7 \mathrm{a}$, the ring down times remained stable, at $1.85 \pm 0.02 \times 10^{-6} \mathrm{sec}$, during the 7 day trial. Based on this result it appears the inlet filter is efficiently removing particulate matter which may have degraded instrument performance. Figure $7 \mathrm{~b}$ depicts the standard deviation of each measured ring down event as obtained by statistical analysis of the entire ring down transient (i.e., the magnitude of the variance of the actual data points about the resulting fit). From Figure $7 \mathrm{a}$ it can be said that the ring down times did not vary much during the 7-day trial and from Figure $7 \mathrm{~b}$ it appears that the data did not become noisier during that same period, as evident by the consistent standard deviation of each ring down event. The relative error (as defined as the standard deviation of each measurement divided by the measurement value) is plotted in Figure 7c. This data shows that the error in each measurement did not increase during the trial (similar to the result presented in Figure $7 b$ ).

\subsubsection{Measurement of Instrument Sensitivity}

Prior to switching to the line monitoring mode (and just after measuring the zero air sample background and SNR) we measured the spectrum associated with a $50 \mathrm{ppmv}$ ammonia/ $\mathrm{N}_{2}$ sample. The peak intensities and the SNR of this spectrum were logged as a function of experiment time. Results of these measurements are shown in Figure 8.

We note that while there is a considerable amount of fluctuation associated with the ammonia mixing ratios as measured using the calibration mixture, there is no long term trend in the data, i.e., no gradual rise or fall in the concentration. This tells us that over the long term, the instrument sensitivity is not drifting. An artificial decrease in concentration is primarily caused by two things; optical alignment and contaminant build up on the cavity mirrors. Measurements of the ring down times associated with the zero-air samples showed that contaminants were not building up on the cavity mirrors. The results of Figures 7 and 8 corroborate this, and also indicate that the CRDS optics are robust enough to maintain their alignment over a 7 day period. 

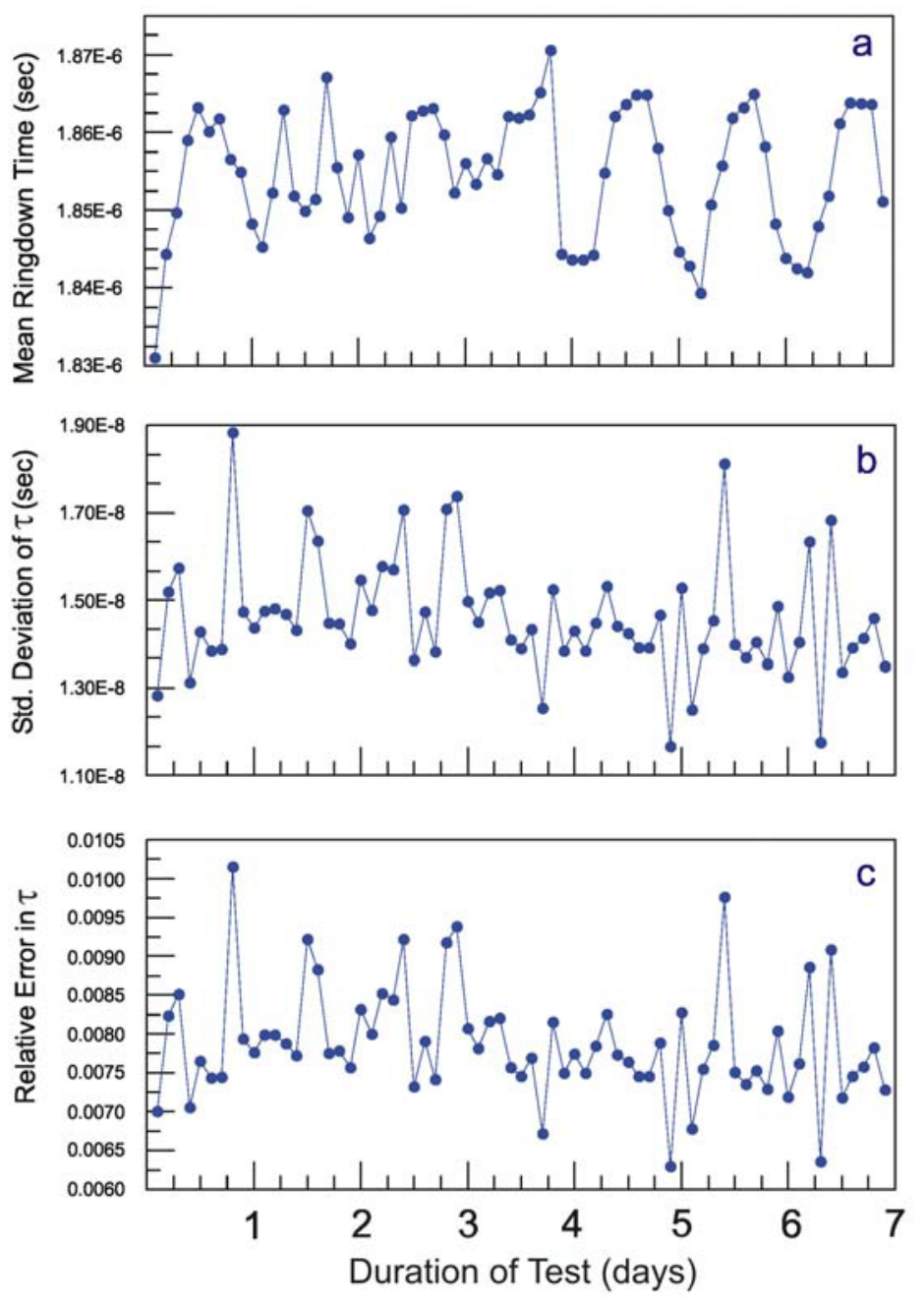

Figure 7. Statistical Analysis of the Ring Down Events During the 7-Day Trial

Since there appears to be no long-term drift in the optical alignment or in the cavity mirror cleanliness we think that the noise associated with the calibrant ammonia concentrations is a manifestation of the relatively low concentration of ammonia present in the calibration mixture which is used in the self-check procedure (i.e., with the $50 \mathrm{ppmv}$ ammonia mixture, the signal to noise ratio in the calibration spectrum is approximately 10). The lower signal to noise ratio in the calibration spectrum results in more scatter being present in the observed ammonia measurements. Even though a $50 \mathrm{ppmv}$ ammonia mixture was used in for the calibration tests shown in Fig. 8, the measured values were typically a factor of two lower. The reason for the disparity is not certain; however, we feel that it is most likely due to a combination of ammonia retention and/or under reported values for the line strengths used to compute concentration. 


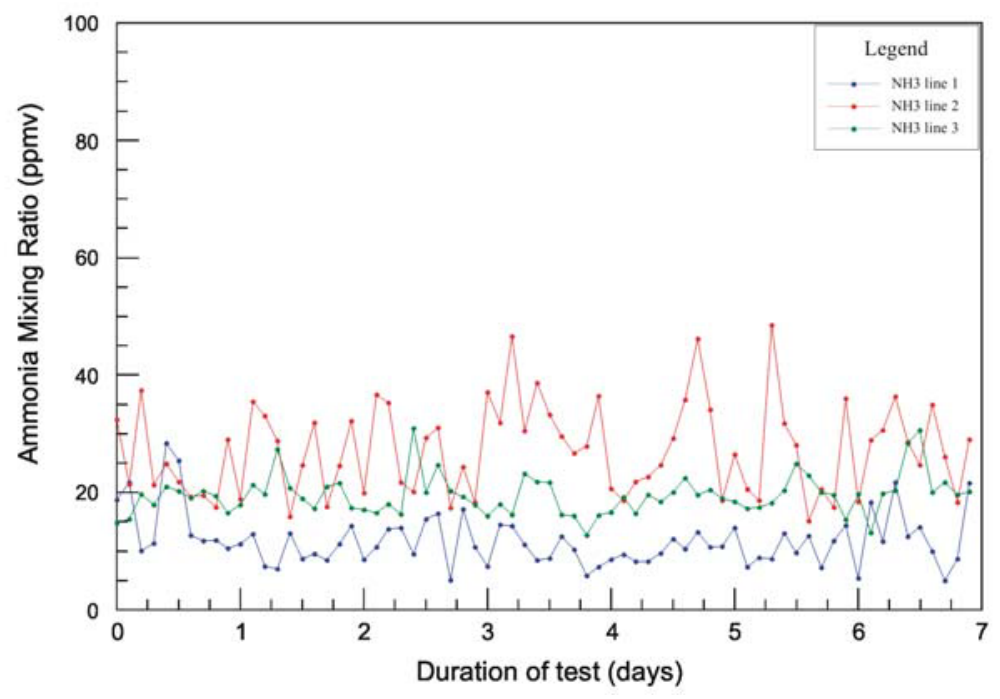

Figure 8. Ammonia Mixing Ratio Obtained from Analysis of Recorded Spectra Containing 3 Distinct Absorption Features as Measured Periodically During 7 Day Trial

\subsubsection{Measurement of Background Variability}

Even though the sensor was operated in a "dirty" environment continuously sampling ambient air, we expected the background signal intensity and noise to match that seen for a zero air sample. This is because the ammonia concentration in ambient air is $\sim 10$ pptv, a level well below our current detection limit. Behavior deviating from this suggests that a) there is far more ammonia in the local air than expected or b) species other than ammonia are contributing to absorption at the monitored wavelength. The two scenarios can be distinguished by switching from line monitoring to spectral monitoring mode. Background variability studies are important to the introduction of any new sensor as it is extremely important to understand which scenarios can lead to false positive detection.

With respect to ammonia, other non-optical ammonia sensors alarm erroneously when exposed to the following species; water, $\mathrm{CO}, \mathrm{NO}_{2}, \mathrm{NO}, \mathrm{SO}_{2}, \mathrm{H}_{2} \mathrm{~S}, \mathrm{Cl}_{2}, \mathrm{H}_{2}, \mathrm{HCN}$, alcohol-based floor cleaning solutions, spray cleaners, contact cleaners, degreasers, paints, silicone sealers (acetic acid), solvent vapors from insulation (urea formaldehyde), methane, combustion products, propane, butane, ethylene, refrigerants, paint vapors (alcohols), smoked meats (nitrates), fruits (ethylene) and cleaning solvents. Based on their spectra, we can immediately rule out the first nine in this list as being potential interferents. To see whether the SWIR CRDS system is insensitive to the other species mentioned we challenged the instrument by exposing samples of their most volatile active ingredients, i.e. methanol, isopropyl alcohol, acetone, acetic acid, hydrogen peroxide, bleach, and aqueous ammonium nitrate, to the instrument inlet nozzle and noting the corresponding change in signal. These experiments were conducted in both the laboratory and in the warehouse. Identical results were seen in both cases.

We saw no interference response from acetic acid, hydrogen peroxide, bleach, aqueous ammonium nitrate or fruits (a banana) when these tests were conducted in the laboratory. We did see a response from methanol, isopropyl alcohol (IPA) and acetone. The latter three species have an extremely broad, but low 
intensity absorption feature in the 1550-1650 nm region (see the section below on high resolution SWIR spectroscopy), and so can be detected by our sensor as is shown in Figure 9.

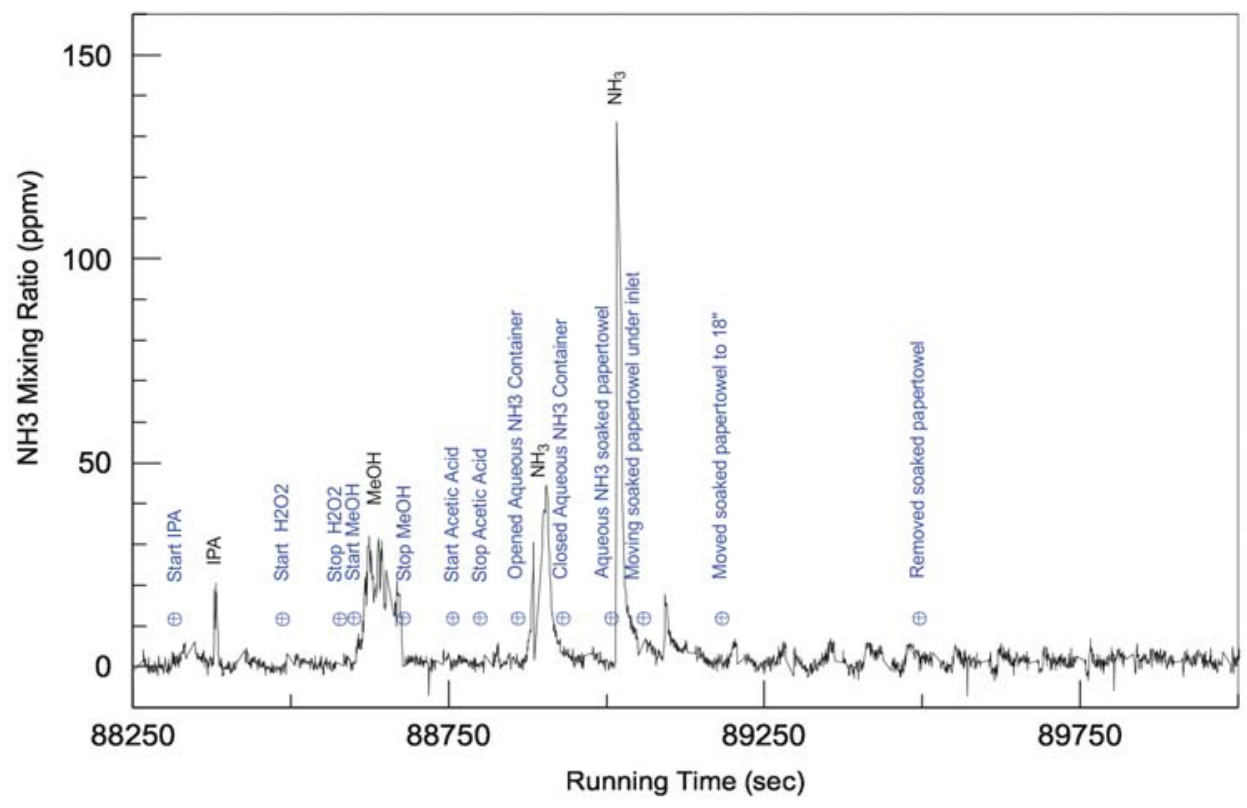

Figure 9. Series of Chemical Challenges. IPA, $\mathrm{MeOH}$ and $\mathrm{H}_{2} \mathrm{O}_{2}$ are isopropanol, methanol and hydrogen peroxide, respectively.

We note that in order to see signal from these species extremely high concentrations must be present. For example if we moved the methanol, IPA, and acetone sample containers 1 foot away from the inlet nozzle, no signal could be discerned. This result suggests that false positive detection can be prevented by keeping potential sources of these chemicals at distances 5 feet or more.

Background variability studies were conducted by operating the sensor in line monitoring mode. The results of experiments done in both the laboratory and in the warehouse, summarized in the plots in Figure 10, indicate that background interference is negligible in these environments as the ambient ammonia concentrations measured over 7 days showed that any variations seen could be attributed to sensor noise, i.e., fluctuations remained within \pm 5 ppmv. Note that the warehouse data is only moderately more noisy (due mainly to temperature drifts of the instrument) than the laboratory data indicating that ambient chemicals found in the warehouse environment, which include diesel exhaust and vapors from packaging materials (isocyanate monomer, etc.) will not cause false positive alarms. We also note that the SWIR CRDS sensor is insensitive to vibration as heavy construction (i.e., jackhammers and backhoes) were in use just outside of the warehouse during part of the time the experiments were being conducted. 

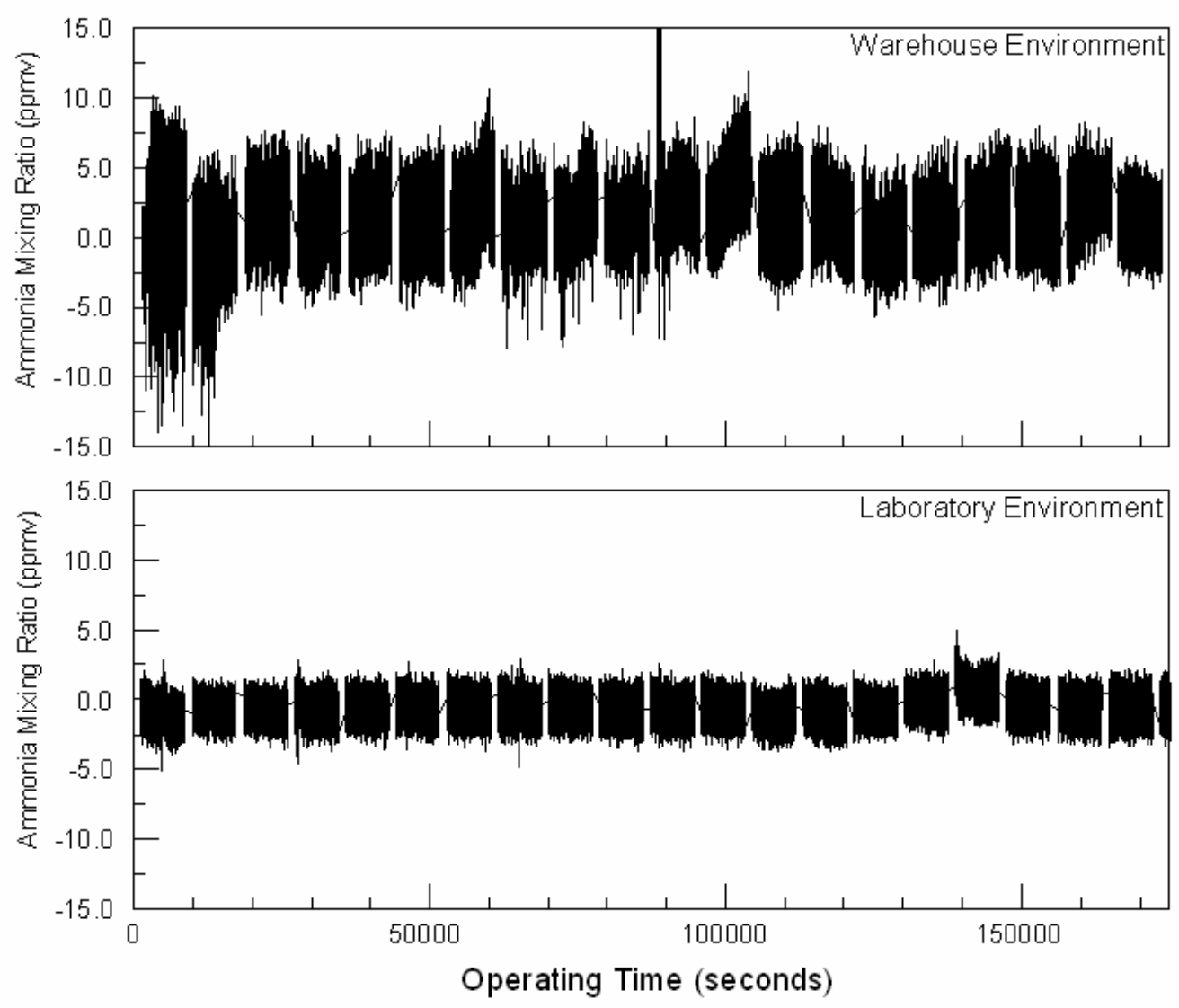

Figure 10. Ammonia Concentration vs. Time for Both the Warehouse and Laboratory Experiments. Plots shown extend over approximately 48 hours. The off-scale spike in the middle of the warehouse data is from our chemical challenge test.

\subsubsection{Conclusion and Future Directions}

When the SWIR CRDS instrument is operated in the line-monitoring mode, the system responds (and gives rise to a signal) whenever any molecule which has an absorption at the laser's wavelength passes through the cell in modest concentrations (i.e., ppmv level). For example, when monitoring for ammonia at $1531.7 \mathrm{~nm}$ methanol produces a signal which is quite strong (as does isopropanol). A rather simple method for resolving this interference is to suspend the line-monitoring mode when a predetermined signal threshold has been crossed and record an absorption spectrum over a small wavelength interval center about the line-monitoring position. If ammonia caused the signal, then any measured absorption spectrum should match the simultaneously recorded ammonia reference spectrum, while if the signal originated from another molecule with a broad featureless profile (e.g., methanol) in the same wavelength region then the recorded spectrum will not match the reference spectrum. For this approach to be successful there are at least two criteria: the sample which gave rise to the original signal must be present in the absorption cell long enough for the instrument to switch over and record a spectrum $(\sim 30 \mathrm{sec}$ to complete) and also the vapor the instrument is configured to monitor must have distinct, resolvable 
absorption features that make it unique. The first criteria could be mitigated somewhat by very quickly (few seconds) closing down the throughput of the gas handling system, thereby retaining the sample in the CRDS absorption cell for a long enough period of time to measure the complete spectrum. The second criteria is more of a challenge as those molecules with broad $\left(>5 \mathrm{~cm}^{-1}\right)$, featureless spectra will be indistinguishable from one another using this technique. Longer spectral scans could be performed if the target molecule's absorption spectrum was sufficiently unique; however the time required to scan over 20-30 $\mathrm{cm}^{-1}$ would be 20-30 times longer than the current mode (i.e., 10-15 minutes per scan), requiring the unknown analyte to be either persistent in the atmosphere or stable in the CRDS absorption cell (if the inlet and outlet were shut after a triggering event).

In future field tests we will use a slightly higher concentration ammonia mixture for our baseline performance measurements (i.e., like the results shown in Figure 8). Presently a 50 ppmv mixture of ammonia in nitrogen is used. Depending on the exact parameters used for the measurements, the signalto-noise ratio of the measured spectra can vary between 10 and 50. If our goal is to study instrument stability then a slightly higher concentration of ammonia (e.g., 200 ppmv in nitrogen) would result in higher signal-to-noise measurements which would be able to show more subtle trends in the data.

\subsection{Feasibility Studies for Sensor Size and Weight Reduction}

\subsubsection{Justification}

Currently, PNNL's SWIR CRD instrument is housed in two separate units. The optical bench ( $2^{\prime}$ x $3^{\prime}$ ) is housed in a travel crate and the supporting instrumentation (i.e., laser power supply, vacuum system, computer and data acquisition, etc.) is housed in a 60" tall instrument rack. This size is consistent with other laser based air sampling sensors created for atmospheric chemistry research. Based on our work to date, we believe there is room for further size reduction. For simplicity and rapid prototype development we were using two computers for data acquisition and gas handling. During FY03 we combined these functions into a single unit allowing us to use a more standard 48 " shippable instrument rack. The next step in size reduction involves placing the entire optical bench within the instrument rack itself. PNNL has been evaluating the use of all-fiber based components for use in SWIR CRD applications and has concluded that dramatic reductions in the size, weight, and cost of the sensor are possible through using individual components and wholly integrated systems originally developed for fiber optic telecommunications applications.

Many of the desired attributes of a telecommunications optical system are the same attributes needed for a sensitive optical sensor. Also, the telecommunications industry has devised a standard frequency grid for the different "channels" used in Dense Wavelength Division Multiplexing (DWDM) which overlap the absorption bands of many smaller molecules. Figure 11 shows a comparison of the absorption spectrum of dilute ammonia taken with PNNL's SWIR CRD instrument along with the International Telecommunications Union (ITU) channels. The upper panel in Figure 11 shows an $85 \mathrm{~cm}^{-1}$ scan of the possible $290 \mathrm{~cm}^{-1}$ of available scanning range of the laser system currently used. A small portion of the spectrum in Figure 11a is expanded and shown in Figure 11b along with markers coinciding with the $50 \mathrm{GHz}$ ITU channels. It is possible to buy inexpensive fiber coupled laser sources designed to operate at each of the specified channels. Additionally, each laser can easily be temperature 
tuned over 2-3 ITU channels. Therefore, it is possible to design an optical sensor to detect one to several chemical compounds, possessing distinct resolvable absorption features near one another, using a single fiber coupled laser source specified for a single ITU channel.
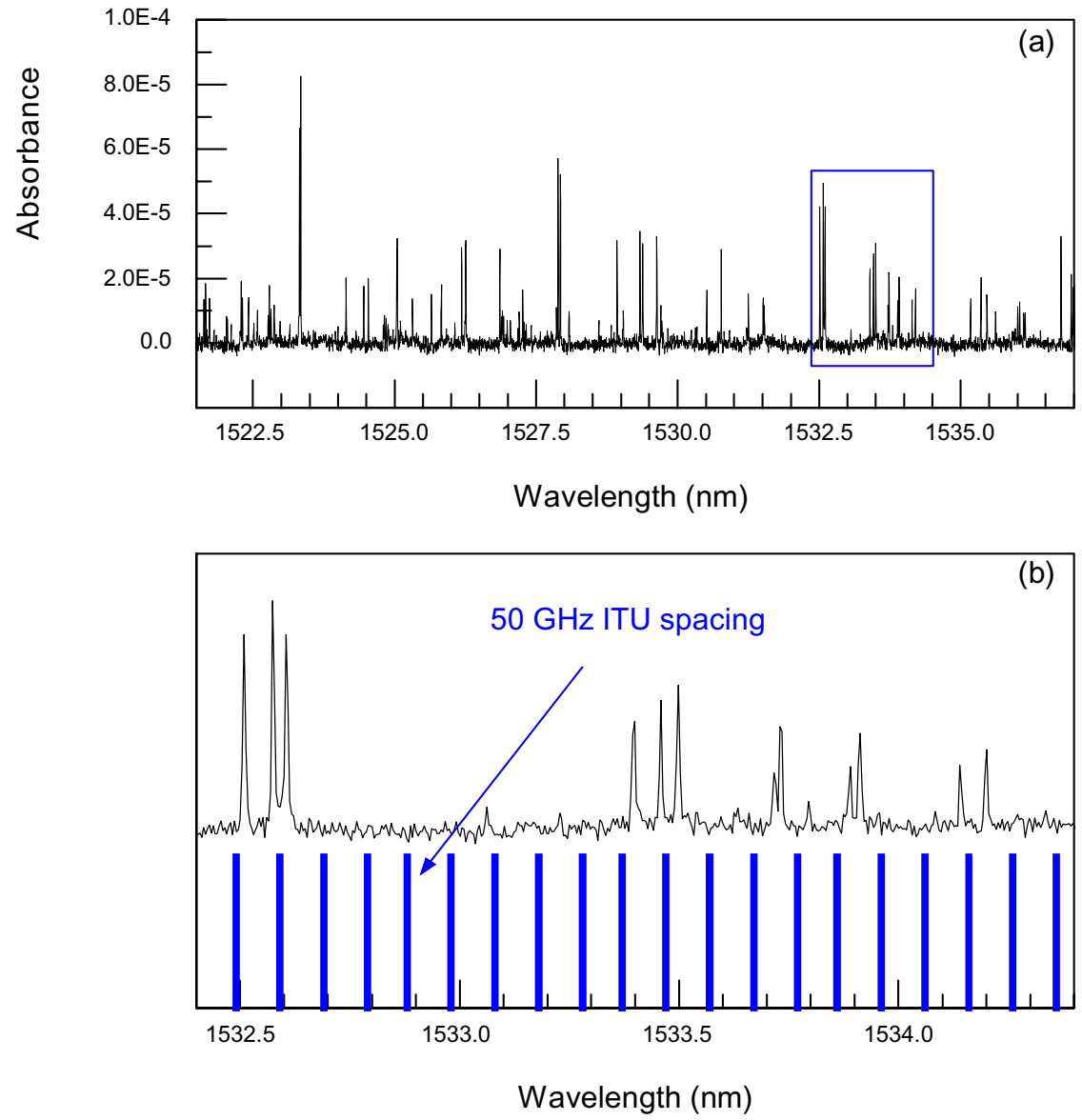

Figure 11. Panel (a) Shows a Portion of the Ammonia Absorption Spectrum Covered by the Diode Laser Used in this Work. Panel (b) shows an expanded region of the spectrum shown in the outlined portion upper panel along with markers showing the $50 \mathrm{GHz}$ ITU channels in this wavelength region.

During FY03 PNNL has evaluated the use of integrated fiber optic coupled laser sources for use in SWIR CRDS. The results are promising, yet technical hurdles exist which preclude the direct replacement of such lasers into our current generation instrument. Presently, the SWIR CRDS instrument incorporates an external-cavity diode laser (ECDL) as the optical source. The ECDL is made up using a standard telecommunication type diode laser combined with an optical grating to produce a system that has wide wavelength tunability. This tunability originates by suppressing the resonator modes of the discrete diode laser (using high-performance anti-reflective coatings) and replacing them with new "external" modes created by proper alignment of an optical grating near the output of the diode laser. By changing the angle of the grating with respect to the diode laser, the output wavelength of the ECDL also 
changes. The external grating not only promotes the broad wavelength tunability, but also acts to stabilize the absolute frequency of the ECDL. This stabilization effect improves the fine wavelength precision of the laser by a factor of 10-100, producing a laser linewidth of roughly $50 \mathrm{kHz}$ (measured over short timescales $<1 \mathrm{~ms}$ ). This is extremely important for our CRDS instrument, since the laser's linewidth is roughly the same order of magnitude as the linewidth of the CRDS optical resonator. This similarity facilitates the optical coupling of the laser light into the cavity used for absorption measurements.

Without the grating to stabilize or reduce the linewidth of the telecommunication type diode laser in the ECDL its linewidth would be a few megahertz. In fact, this broad linewidth has been observed in the standard miniature fiber-coupled laser diodes which we have investigated for size reduction purposes. The impact that this increased linewidth has on system performance is dramatic, as the coupling efficiency between the laser and cavity decreases as linewidths become dissimilar. As an analogy, it is similar to trying to thread a needle after having had several cups of coffee, your hand (i.e., the laser's frequency) is moving too quickly to insert the thread into the eye (the cavity's acceptance linewidth). This is not an insurmountable problem but it is a technical hurdle. Our approach will be to actively stabilize the laser source and cause its frequency to track that of the cavity for the very short amount of time needed for efficient coupling. In order to implement this feature only one additional optic is needed (a quarter waveplate) and one additional detector to measure an error signal that is generated when the laser and cavity are not in resonance. This error signal will be used to force the laser's frequency to track the cavity resonance (only for a few microseconds).

Integrated telecommunications systems (the size of a palm pilot) contain nearly all of the required electro-optical components necessary for a CRD system. The digital photograph shown in Figure 12 depicts a unit manufactured by JDS Uniphase for use as an optical transmitter for a specific ITU channel near $1535.03 \mathrm{~nm}$. It contains a $50 \mathrm{~mW}$ laser source, three monitoring photodiodes, two beam splitters, an intensity modulator capable of switching times less than $10 \mathrm{nsec}$, and an integrated wavelength locking module for precise wavelength control. The cost of these surplus units is currently less than $\$ 1 \mathrm{~K}$, which indicates that CRD sensor costs of less than $\$ 5 \mathrm{~K}$ are possible. PNNL will continue to evaluate the suitability of these units for CRD spectroscopy in FY04, and possibly develop a prototype instrument for demonstration. 


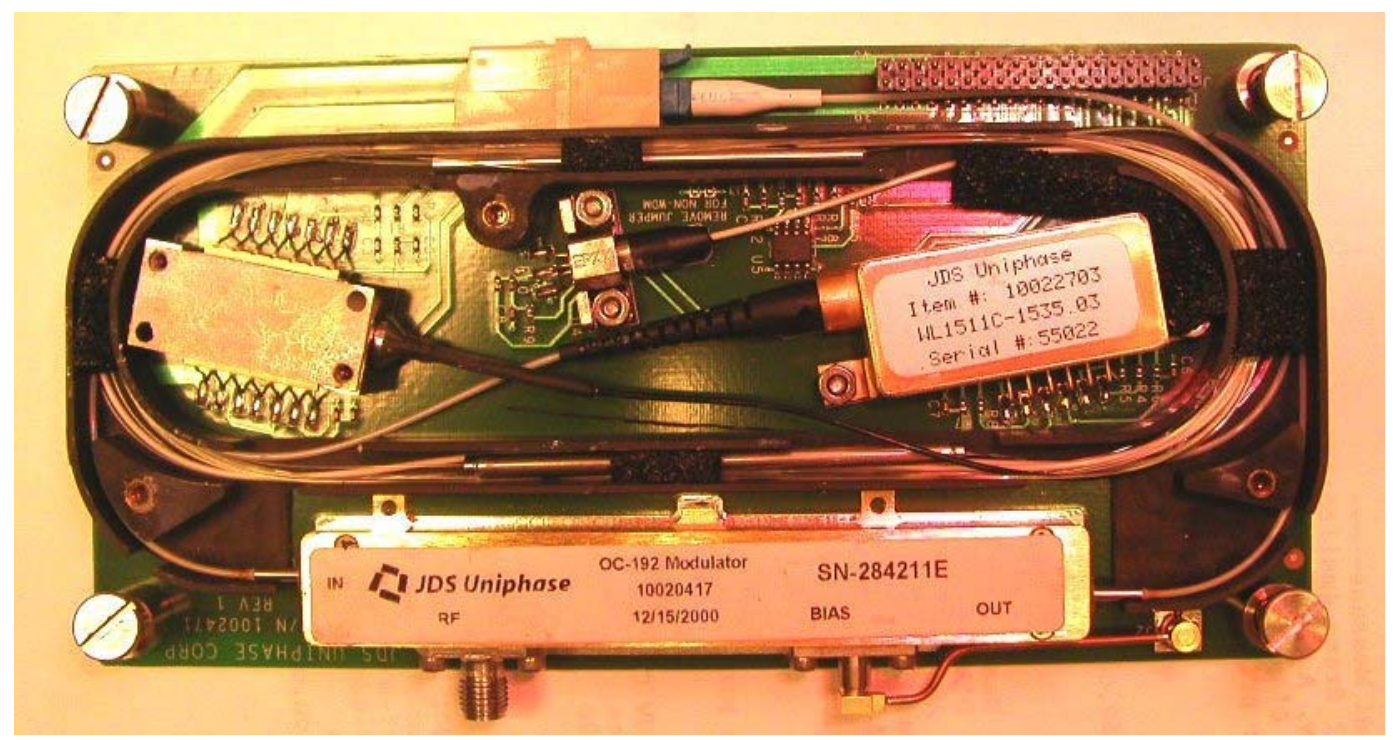

Figure 12. SWIR Fiber-Optic Transmitter Module for Digital Optical Fiber Networking Proposed as Optical Unit For Micro-CRD System. Upper left is a $50 \mathrm{~mW}$ laser source, upper right is a wavelength locking module for a specific ITU channel at $1535.03 \mathrm{~nm}$, bottom is a $10 \mathrm{Gbit} / \mathrm{s}$ lithium niobate intensity modulator. The entire unit is 3 " $\mathrm{x} 6$ " and weighs about $14 \mathrm{oz}$.

\subsubsection{Generation I All Fiber SWIR CRDS}

A prototype optical fiber based SWIR CRD instrument was assembled and early tests were performed. A fiber optic coupled optical switch (acousto-optic modulator) was tested and its performance is excellent, with a measured switching time of $<100 \mathrm{nsec}$. The laser source used was a fiber coupled laser used for telecommunications applications. It has a higher output power than the external cavity diode laser (ECDL) currently used in the SWIR CRD instrument (60 $\mathrm{mW}$ versus $10 \mathrm{~mW}$ ). The spatial quality, and hence the optical coupling to the CRD absorption cavity, is also superior as the light is coupled via a single-mode optical fiber that only allows a purely circular Gaussian mode to propagate, as opposed to the diverging, elliptical beam from the ECDL. A schematic of the current prototype system is shown in Figure 13. It consists of a standard telecommunications laser emitting at $1535 \mathrm{~nm}$ through a single-mode fiber output. This output is directly coupled to the input of the acousto-optic modulator (AOM) switch and then coupled to the Fabry-Perot cavity via a free-space coupling lens. 


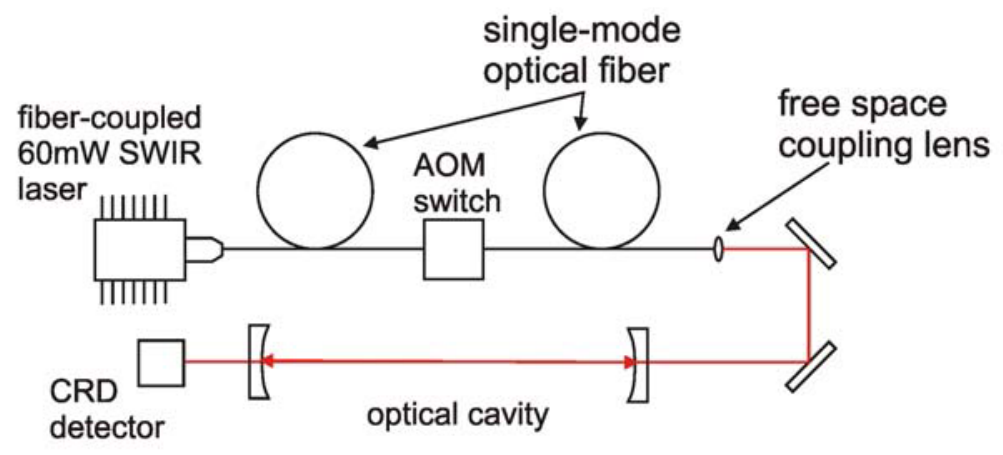

Figure 13. Schematic Representation of SWIR Fiber-Optic Based CRD Instrument

A photograph of the fiber SWIR CRD system is shown in Figure 14 with a ruler to give a sense of scale. One of the first ring down traces measured with this instrument is shown in Figure 15. Further development of the system will continue in FY04.

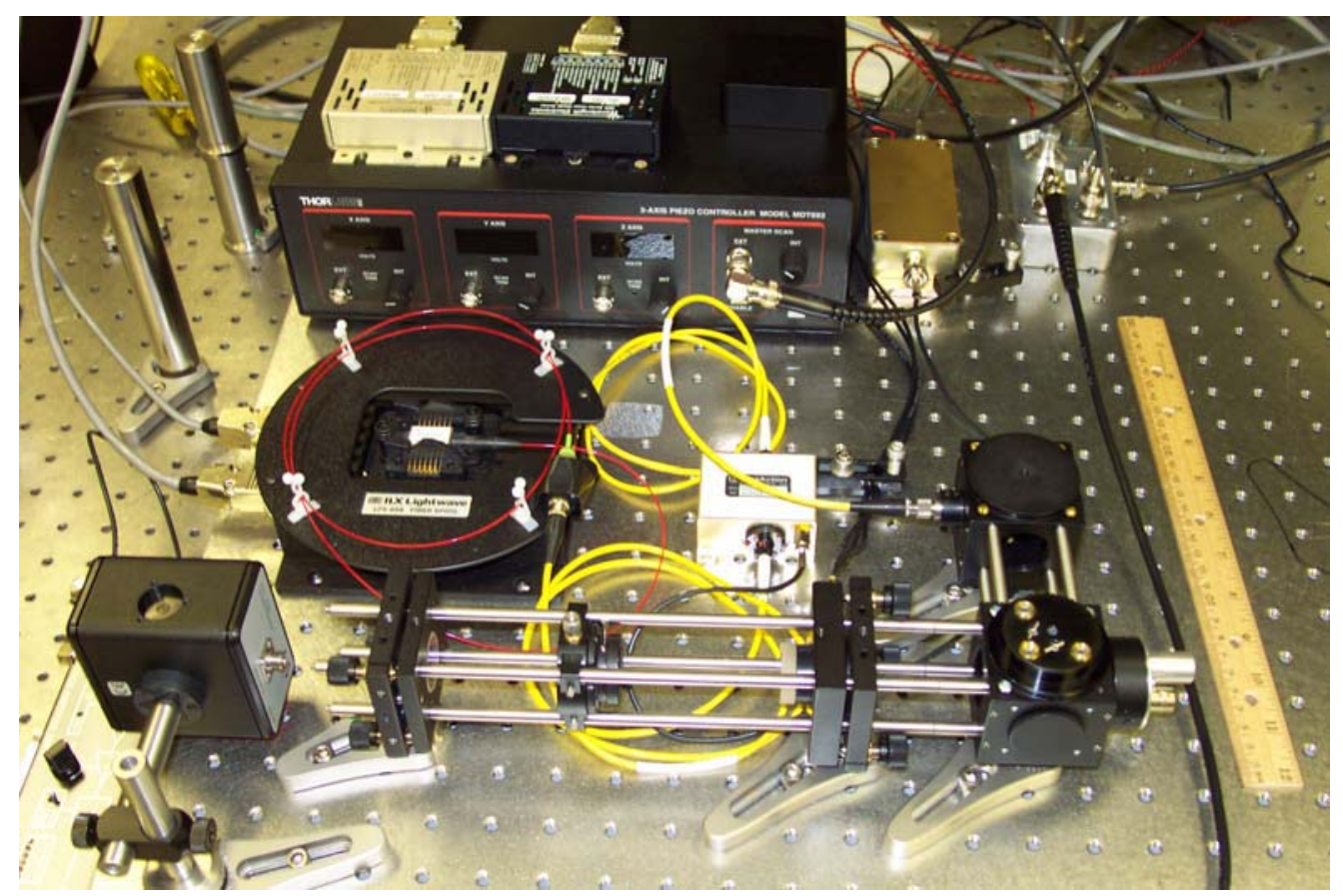

Figure 14. Digital Photograph of All-Fiber SWIR CRDS Prototype. A 12" ruler shows the compact layout of the instrument. 


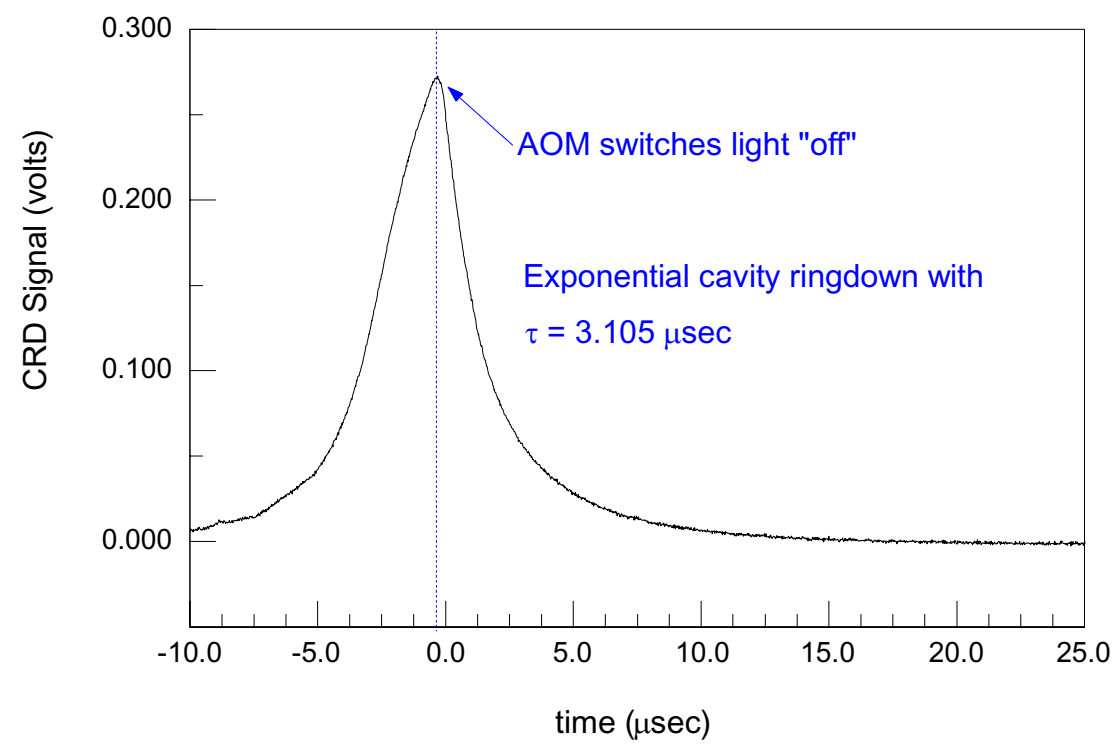

Figure 15. Early Cavity Ring Down Trace from Fiber CRD Instrument

\subsection{High Resolution SWIR Spectroscopy of WMD-related Molecules}

To expand the number of possible chemicals the SWIR CRD instrument can detect it is necessary to collect information on the SWIR absorption spectra of many proliferant chemicals. The requirements for these spectra are unique as they need to be obtained under the conditions used in the CRDS, i.e., at reduced pressure and slightly elevated temperature. Most IR spectra, including those in the PNNL/DOE IR database, are recorded at atmospheric pressure and emphasize the mid- to long-wave IR region. Initial searches of the scientific literature have resulted in very few published spectra for compounds related to chemical weapons or explosives. The few spectra which do exist have not been recorded with adequate spectral resolution.

Part of our FY03 activities in this area have focused on identifying species associated with WMD proliferation and CWA production that are amenable to detection using CRDS. We have modified our Bomem DA3 Fourier transform infrared (FTIR) spectrometer by adding a 3 meter, fixed-pathlength absorption cell and an indium gallium arsenide SWIR detector. This instrument is capable of measuring SWIR spectra in the 5000 to $10000 \mathrm{~cm}^{-1}$ range with $0.02 \mathrm{~cm}^{-1}$ resolution. This resolution, $600 \mathrm{MHz}$, is roughly equivalent the Doppler width of SWIR molecular transitions. We have collected data on ammonia and methanol, and Figure 16 shows a representative spectra of methanol. 


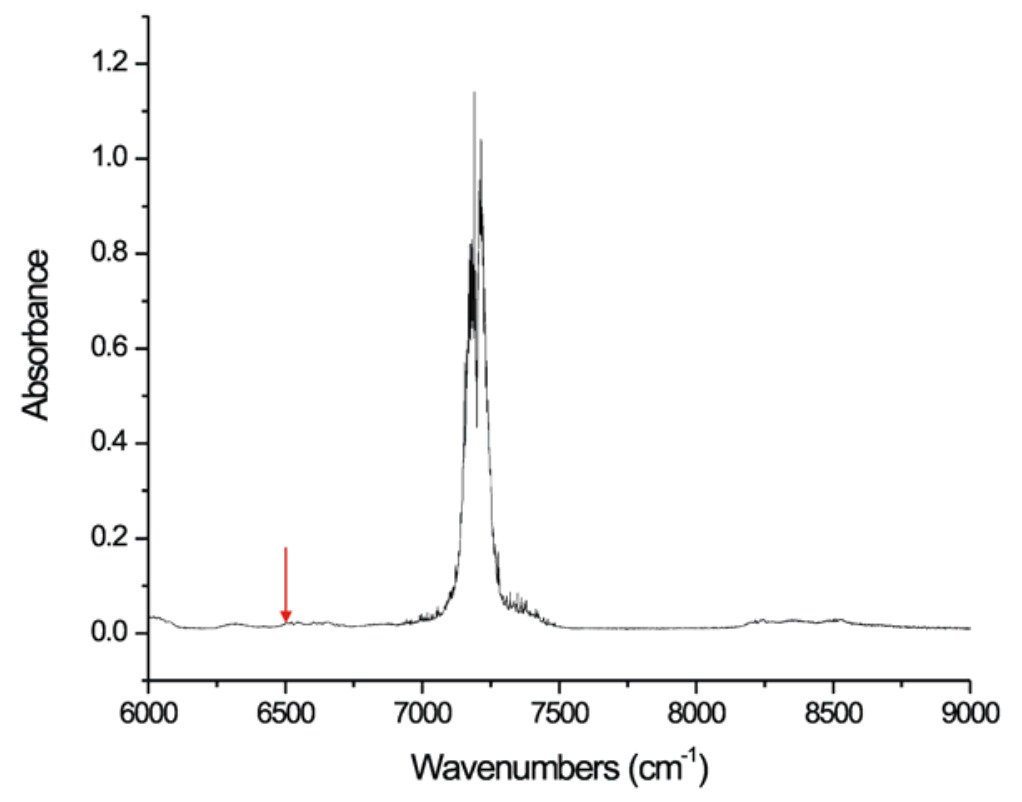

Figure 16. Short-Wave Infrared Absorption Spectrum of Methanol at Low Pressure Using the Modified Bomem FTIR. The red arrow shows the spectral position where the SWIR CRDS system was set to detect ammonia.

\subsection{FY04 Research Plans}

The proposed plan for the SWIR CRD project at PNNL in FY04 includes the following areas of emphasis: 1) continued field testing of the generation I sensor, 2) CRDS sensitivity improvement by incorporating better mirrors in the cavity, 3) continued building and testing of generation II all-fiber SWIR CRDS sensor, 4) gathering data concerning the SWIR spectroscopy of relevant molecules, and 5 ) identify external missions and clients for the SWIR CRDS sensor. We discuss these activities in more detail below.

\subsection{Field Tests}

In FY04 we will test the first generation SWIR CRDS in several different ambient air environments to prove that the system can operate stably and independently over long periods with minimal operator assistance. We will also collect information on background variability, instrument sensitivity and signal to noise drift during these tests. Four or more tests will be conducted in a variety of locations that include PNNL's shipping and receiving building, a large indoor facility such as the Pasco airport, a minimally polluted outdoor air environment such as the parking lot at 2400 Stevens, and in an outdoor vehicle queue to mimic a border crossing environment. Fourier-transform spectrometer based environmental monitoring will be conducted simultaneously so that we can attempt to identify which chemicals are present in the ambient environment so that we can quantify the SWIR CRDS sensor selectivity, and accurately identify which species, if any, act as interferents. We note here that the frequency of 
background interference seen during these tests will determine if changes in the signal monitoring procedure, which were outlined in a previous section, need to be permanently implemented. The data collected on background variability and system performance will be handed over to the System Performance Analysis team.

\subsection{CRDS Sensitivity Improvement}

Preliminary results of a SWIR CRDS experiment done in FY03 using higher reflectivity mirrors, producing a higher finesse optical cavity, show that several improvements need to be made before we can utilize these devices to improve the technique's ultimate detection limit. The discussion of these results is identical to the discussion made in Section 3.1.4 above concerning the mismatch of laser linewidth with cavity linewidth. In the case described earlier the fiber-coupled laser source had a broader linewidth than our existing cavity linewidth resulting in reduced performance. In the case where higher reflectivity mirrors were inserted into the CRD instrument, the cavity linewidth was decrease by a sufficient amount that the already narrow linewidth of the external-cavity diode laser was too broad, resulting in the same degradation of performance. Again, the use of a transient-locking scheme can be used to control the frequency of the source laser just long enough to facilitate laser/cavity resonance and improve performance.

We will also conduct a detailed study on optical cavity noise sources and their influence on long-term stability and precision. The results of this study will allow a greater understanding of the mechanisms that lead to variations in cavity ringdown times (i.e., intra-cavity photon lifetime). Drifts in the baseline ringdown times lead to slow variations in instrument accuracy. A preliminary review of the literature suggests that subtle spatial loss mechanisms (primarily from intra-cavity diffraction) may play an important role in determining baseline stability. The ultimate detection limit of any cavity ringdown spectrometer is limited by the ability of the instrument's system to measure cavity loss accurately and precisely. Presently our instrument is capable of discerning relative differences in single-pass loss on the order of 1 part-per-million. Another way of saying that is the instrument can register a change in the ringdown time constant which corresponds to change in the photon lifetime inside the cavity on the order of 1 ppmv. This is in fact what gives the CRDS its ability to make such sensitive measurements of absorbers in the cavity. However, the baseline value does not stay stable for any extended period of time. The "empty" cavity ringdown time constant can vary by up to $1 \%$ or more depending on the conditions and the duration of the measurement. Based on these observations, we will attempt to further understand the origins of such variations and their statistical impact on the data recovery with the goal of minimizing such impacts and hence improving the minimum detection levels of species of interest.

The results of these experiments will also allow us to specify the external laser cavity stability requirements that the LWIR Quantum Cascade Laser (QCL) team will need for building their components.

\subsection{Generation II All-fiber SWIR CRDS}

As mentioned above the use of fiber-coupled telecommunication type diode lasers and other fiber based components will continue to be evaluated for their suitability in a smaller SWIR CRDS system. 
Again the focus will be on improving the instantaneous resonance condition which must exist between the laser source and the cavity for there to be efficient optical coupling between the two.

\subsection{High Resolution SWIR Spectroscopy of Relevant Molecules}

As the PNNL Signatures Team continues to develop information about molecules relevant to DOE's proliferation detection mission we will ascertain whether SWIR spectra exist for those molecules, and if they do not we will decide if the spectra should be recorded. Molecules which are likely to be investigated include nitro-benzene and nitro-toluene (as they are relevant to explosives detection) and dimethyl methyl phosphonate (as it is often used as a chemical agent simulant).

\subsection{External Client Development}

Our last mission in FY04 is to identify external missions and clients for SWIR CRDS monitoring and to determine the CRDS system specifications, i.e., target the laser, sampling system and software needed to detect the species at the concentration levels required by the client. 


\section{Distribution}

No. of

\section{Copies}

\section{OFFSITE}

Mr. W. Randy Bell

United States DOE

NNSA/NA-22

1000 Independence Ave. SW

Washington, DC 20585

Dr. David Berry

United States DOE

NNSA/NA-22

1000 Independence Ave. SW

Washington, DC 20585

LTC John C. Carrano, PhD

Program Manager

DARPA, MTO

3701 N. Fairfax Dr.

Arlington, VA 22203-1714

Mr. Ralph Hastings

United States DOE

NNSA/NA-22

1000 Independence Ave. SW

Washington, DC 20585

Mr. Eric Sanders

United States DOE

NNSA/NA-22

1000 Independence Ave. SW

Washington, DC 20585
No. of

Copies

Dr. Vaughn Standley

United States DOE

NNSA/NA-22

1000 Independence Ave. SW

Washington, DC 20585

\section{ONSITE}

\section{Pacific Northwest National Laboratory}

$\begin{array}{ll}\text { Aker, PM } & \text { K5-25 } \\ \text { Cannon, BD } & \text { K5-25 } \\ \text { Doherty, TJ } & \text { K8-09 } \\ \text { Harper, WW } & \text { K5-25 } \\ \text { Johnson, BR } & \text { K6-24 } \\ \text { Schultz, JF (26) } & \text { K5-25 } \\ \text { Sheen, DM } & \text { K5-25 } \\ \text { Stewart, TL } & \text { K5-25 } \\ \text { Taubman, MS } & \text { K5-25 } \\ \text { Thompson, JS } & \text { K5-25 } \\ \text { Williams, RM } & \text { K5-25 } \\ \text { Information Release Office (7) } & \text { K1-06 }\end{array}$

